\title{
Beiträge zur Kenntnis der cavernicolen Antroleucosomiden (Diplopoda, Ascospermophora)
}

\author{
Von Ionel Tabacaru ${ }^{1}$ )
}

Mit Tafel 1(1)-13 (13)

\begin{abstract}
Herrn Prof. Dr. C. Мотаş zum 75. Geburtstag in Verehrung
\end{abstract} und Dankbarkeit gewidmet

Die aus der östlichen Hälfte der Balkanhalbinsel und den Südkarpaten bekannten cchten, höhlenbewohnenden Ascospermophoren gehören ausschließlich der Familie Antroleucosomidae an. So ist aus Höhlen im Westen der Südkarpaten (Rumänien) die Gattung Antroleucosoma mit 2 Arten, A. (A.) banaticum Verh. 1899 und A.(Heteroantroleucosoma) spelaea Ceuca 1964, bekannt und aus Höhlen Ostserbiens und Bulgariens die Gattung Bulgarosoma mit den Arten B.bureschi Verh. 1926, B.crucis Strass. 1960 und B.rhodopinum Strass. 1966. Ebenfalls aus Bulgarien ist noch eine höhlenbewohnende ProdicusArt, Prodicus (Balkandicus) albus Strass. 1960, bekannt.

Anläßlich der gemeinsam mit meinem Kollegen und Freund Dan Dancău in den Südkarpaten und Banatergebirge vorgenommenen biospeläologischen Forschungen, wurden 2 neue höhlenbewohnende Antroleucosomiden entdeckt, und zwar eine Art, die einer neuen Gattung angehört, Dacosoma motasi n.g., n.sp., sowie eine neue Art der Gattung Bulgarosoma. Ein reiches Material von dieser neuen BulgarosomaArt wurde mir nachträglich von meinen Kollegen A. und Şt. Negrea zur Verfügung gestellt.

Gelegentlich einer internationalen speläologischen, im Rhodopegebirge von der bulgarischen touristischen Gesellschaft organisierten Expedition, welche von den Prof. P. Tranteev aus Sofia und D. Raitcheff aus Tchepelare geleitet wurde, haben Prof. Tr. Orghidan und meine Kollegin Frau Anca Burghele-Bălăcescu Ascospermophoren-

1) Institutul de Speologie, Str. Gutenberg Nr. 3, Bucuresti, Romania. 
Material gesammelt, das zu einer neuen Bulgarosoma-Art gehört, die ebenfalls hier beschrieben wurde.

Desgleichen hatte ich die Möglichkeit, die Beschreibung der Arten Antroleucosoma banaticum Verh. 1899 und Bulgarosoma bureschi Verh. 1926 zu ergänzen, auf Grund der Untersuchung von Material aus Höhlen, aus denen sie ursprünglich beschrieben wurden.

Allen, die mir für diese Arbeit Material zur Verfügung stellten, möchte ich auch an dieser Stelle bestens danken. Für viele wertvolle Ratschläge möchte ich meinen herzlichsten Dank dem bekannten Diplopodenspezialisten und Höhlenforscher Herrn Karl Strasser (Triest) aussprechen.

\section{Familie ANTROLEUCOSOM I DAE Verh. 1909}

Im Jahre 1899 beschrieb K. W. Verhoeff einen neuen Diplopoden aus einer Höhle der Südkarpaten, für den er die Gattung Antroleucosoma und die Sippe Antroleucosomini innerhalb der Unterfamilie CraspedosominaeFamilie Chordeumidae festlegte. Der gleiche Autor gruppierte im Jahre 1900 die bis dahin bekannten Ascospermophoren in 3 Familien, Chordeumidae, Craspedosomidae und Trachysomidae, und legte für die Gattungen Antroleucosoma Verh. 1899 und Prodicus Attems 1899 die Unterfamilie Antroleucosominae innerhalb der Familie Craspedosomidae fest. Im Jahre 1909 gibt Verhoeff ein neues System der Diplopoda Ascospermophora, in dem er 14 Familien unterscheidet, darunter auch die Familie Antroleucosomidae.

1914 stellte Verhoeff in die Familie Antroleucosomidae, aber in einer gesonderten Unterfamilie Anthogoninae, auch die Gattungen Anthogona Ribaut 1913 und Cranogona Ribaut 1913, die H. Ribaut zusammen mit 2 anderen Gattungen (Scutogona und Origmatogona) in die Familie Anthogonidae Ribaut 1913 einbezogen hatte. Später fügte Verhoeff (1926 b) den beiden Gattungen der Unterfamilie Antroleucosominae, Antroleucosoma und Prodicus die Gattung Bulgarosoma Verh. 1926 hinzu, die für eine neue Art aus einer Höhle der westlichen Balkangebirge aufgestellt wurde. Derselben Unterfamilie gliedert Verhoeff auch die Gattung Acherosoma Verh. 1929 an, welche er für die von R. Latzel (1884) unter dem Namen Craspedosoma (Scotherpes) troglodytes aus der Höhle Postoina beschriebene Art aufstellte.

Im Jahre 1930 teilt Verhoeff die Familie Antroleucosomidae in 3 Unterfamilien ein: 1. Antroleucosominae mit den Gattungen Antroleucosoma, Prodicus und Bulgarosoma; 2. Anthogoninae mit den Gattungen Anthogona und Cranogona; 3. Acherosominae mit der Gattung Acherosoma. In dem Band Diplopoda in Bronns Klassen und Ordnungen des Tierreichs jedoch veröffentlicht Verhoeff ein neues System der Ascospermophora, in dem er die Familien in Superfamilien und Cohorten gruppiert. In dieser neuen Klassifikation werden die Gattungen Anthogona und Cranogona in eine gesonderte Familie, Anthogonidae, gruppiert, die zusammen mit der Familie Antroleucosomidae die Superfamilie Haplomera bilden.

Später wurden sodann noch weitere, zahlreiche Arten und Unterarten der Gattungen Prodicus und Acherosoma beschrieben. Desgleichen wurden (Verhoeff 1940 a und b) innerhalb der Gattung Prodicus die Untergattungen 
Paraprodicus Verh. 1940 für die Art P. penicillatus Attems 1902 und Hellasdicus Verh. 1940 für die Art P. penteliconus Verh. 1925 festgelegt; die Gattung Acherosoma wurde in 2 Untergattungen, Acherosoma s. str. und Macrotelosoma Strasser 1935, geteilt.

In einer posthum erschienenen Arbeit (1959) über die Myriopoden aus den Höhlen der Balkanhalbinsel legt C. Attems ein neues System der Ascospermophoren fest, in dem er eine Reihe von Änderungen an dem von Verhoeff eingeführten System vornimmt. In bezug auf die Antroleucosomidae möchten wir hervorheben, daß Attems die Gattung Acherosoma aus der Familie Antroleucosomidae in die Familie Anthogonidae überträgt und im Rahmen der Superfamilie Haplomera neben die beiden erwähnten Familien auch die Familie Heterolatzelidae einführt.

1960 beschreibt K. Strasser eine neue Gattung aus der Familie Antroleucosomidae, und zwar Bulgardicus für eine neue Art aus einer Höhle des Balkangebirges sowie eine neue troglobionte Art der Gattung Bulgarosoma aus dem östlichen Serbien. Gleichzeitig trennt Strasser die Untergattung Paraprodicus Verh. von der Gattung Prodicus Attems als eine separate Gattung und unterscheidet innerhalb der Gattung Prodicus 2 weitere Untergattungen, Osmandicus für die Arten P. bilselii Verh. 1940 und P. halophilus Verh. 1940 und Balkandicus für eine neue Art aus einer Höhle des Balkangebirges.

1964 beschreibt T. Ceuca eine neue Antroleucosoma-Art aus einer Höhle der Südkarpaten und stellt für diese die neue Untergattung Heteroantroleucosoma auf. Derselbe Autor beschreibt (1964 b) in einer Arbeit über die Höhlendiplopoden Jugoslawiens (Sammlung Biospeologica) eine neue Unterart von Bulgarosoma crucis Strass. 1960, die ebenso aus einer Höhle Ostserbiens stammt.

In einer vor kurzem erschienenen umfangreichen Arbeit über die Diplopoden Bulgariens beschreibt Strasser (1966) auch eine neue BulgarosomaArt aus einer Höhle in Zentral-Rhodopegebirge, B. rhodopinum.

Aus obigen Ausführungen geht hervor, daß bei Anerkennung einer gesonderten Familie Anthogonidae Ribaut 1913, die nicht nur die Gattungen Anthogona und Cranogona, sondern auch die Gattung Acherosoma umfaßt, die Familie Antroleucosomidae Verh. 1909 mit folgenden Gattungen verbleibt: Antroleucosoma Verh. 1899 (mit den Untergattungen Antroleucosoma s. str. und Heteroantroleucosoma Ceuca 1964), Prodicus Attems 1899 (mit den Untergattungen Prodicus s. str., Hellasdicus Verh. 1940, Osmandicus Strass. 1960 und Balkandicus Strass. 1960), Bulgarosoma Verh. 1926, Paraprodicus Verh. 1940 und Bulgardicus Strass. 1960.

In Anbetracht dieser Gattungen kann die Familie Antroleucosomidae durch folgende morphologische Merkmale gekennzeichnet werden:

30 Segmente, Rumpfringe mit Seitenwülsten und auf jeder Seite 3 Makrochaeten. Antennen schlank. Gnathochilarium mit Promentum. Beinpaare ohne Tarsalpapillen; 1. und 2. Beinpaar mit tarsalem Borstenkamm. Keine selbständigen Penes; Vasa deferentia münden in die Hüften des 2. Beinpaars. 3.-7. Beinpaar des $\widehat{\jmath}$ meistens verdickt 
und mit Modifizierungen (Ausnahme nur Antroleucosoma banaticum). 8. und 9. Beinpaar des ơ mit Coxalsäcken, 9. Beinpaar mit 1 oder 2 Coxitfortsätzen. Keine Nebengonopoden. Vordere Gonopoden, ohne Cheiritbildung, mit stark verwachsenem und teilweise einheitlichem Syncoxit, welcher keinen Grannenapparat besitzt. Hinteres Gonopodensegment bildet meistens ein Podosternit mit 1 unpaaren medianen oder 2 paramedianen Fortsätzen (Ausnahme nur Bulgardicus). Die Telopodite der hinteren Gonopoden sind mehr- bis weniggliedrige, gerade, distal gerichtete Stummel oder ungegliederte Reste oder gänzlich fehlend.

Durch die morphologischen Merkmale ihrer Vertreter stehen sich die Familien Antroleucosomidae und Anthogonidae sehr nahe, so daß uns ihre Gruppierung in eine gesonderte Superfamilie, Haplomera Verh., als gerechtfertigt erscheint. Jedoch sind wir der Meinung, daß die Einbeziehung der Familie Heterolatzelidae in diese Superfamilie nicht angebracht ist. Dies in Anbetracht dessen, daß die Vertreter dieser Familie an dem 3.-7. Beinpaar Tarsalpapillen aufweisen.

\section{Bestimmungsschlïssel für die Gattungen der Familie Antroleucosomidae}

Auf der Grundlage des von Verhoeff (1932) festgelegten Bestimmungsschlüssels für die Gattungen der Unterfamilie Antroleucosominae beziehungsweise Bulgarosoma, Antroleucosoma und Prodicus und insbesondere gestützt auf den von K. Strasser (1960) festgelegten Bestimmungsschlüssel, in dem die Gattungen Paraprodicus, Bulgarosoma, Bulgardicus und Prodicus berücksichtigt wurden, geben wir in der Folge einen neuen Bestimmungsschlüssel für alle bisher bekannten Gattungen der Familie Antroleucosomidae, die neue, hier beschriebene Gattung Dacosoma mit inbegriffen.

1 (2) Vordere Gonopoden gänzlich ohne Telopodite, nur mit einem syngonopodialen Aufsatz, doppelt so breit wie hoch, welcher vorn in der Mediane 2 lange und schlanke, nach außen gebogene Fortsätze trägt. Hintere Gonopoden ohne mediane Sternitfortsätze, nur mit einheitlichen gedrungenen, in der Mediane zusammenstoßenden Coxiten, welche am äußeren Endrand Telopoditrudimente tragen.........

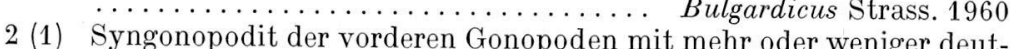
. lichen Seitenteilen (Telopoditen) und mindestens ebenso hoch wie breit. Hintere Gonopoden mit einem medianen unpaarigen Sternitfortsatz oder 2 paramedianen Fortsätzen, getrennt von den äußeren gonopodialen Fortsätzen, welche meistens Telopoditrudimente tra-

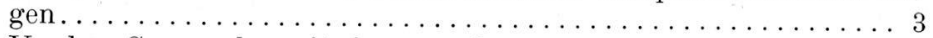

3 (4) Vordere Gonopoden mit einem großen, medianen, unpaaren Aufsatz, welcher vorn in ein Triangulum erweitert ist. Podosternit des hinteren Gonopodensegments in der Mediane mit 2 weit voneinanderstehenden Fortsätzen ............ Bulgarosoma Verh. 1926

4 (3) Vordere Gonopoden ohne medianen unpaaren Aufsatz, in ein Triangulum erweitert. Podosternit des hinteren Gonopodensegments 
mit einem medianen unpaaren Fortsatz oder 2 nahe beieinander-

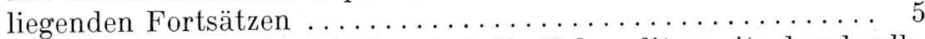

5 (6) An den vorderen Gonopoden sind die Telopodite weitgehend selbständig geblieben und von dem zentralen syncoxalen Abschnitt durch tiefe Bucht getrennt .......... Paraprodicus Verh. 1940

6 (5) An den vorderen Gonopoden sind die Telopodite mit dem zentralen syncoxalen Abschnitt mehr oder weniger verwachsen . . . . . . 7

7 (8) Podosternit des hinteren Gonopodensegments mit 2 paramedianen, selten teilweise miteinander verwachsenen Fortsätzen. Körper meistens pigmentiert und stets mit zahlreichen Ocellen ...........

Prodicus Attems 1899

8 (7) Podosternit des hinteren Gonopodensegments mit einem unpaaren Medianfortsatz. Pigmentlos und blind .............. 9

9 (10) Vordere Gonopoden mit pseudoflagelloiden Fortsätzen; die vorderen blattartigen Gonocoxite sind bis zu ihrer Basis untereinander getrennt. Hinteres Gonopodensegment mit einem breiten Sternitfortsatz, mit dem die Coxitfortsätze verschmolzen sind; Telopodite der hinteren Gonopoden als winzige Rudimente seitlich auf einem Femoroid sitzend ................Antroleucosoma Verh. 1899

10 (9) Vordere Gonopoden ohne pseudoflagelloide Fortsätze; die vorderen blattartigen Gonocoxite sind im basalen Teil zu einem gemeinsamen schmalen Stamm verschmolzen. Hinteres Gonopodensegment mit einem schmalen medianen Sternitfortsatz getrennt von den häutigen Coxitfortsätzen; Telopodite der hinteren Gonopoden sind mehrgliedrige, gerade, distal gerichtete Stummel ..... Dacosoma n.g.

Dacosoma n.g.

\section{Diagnose:}

30 Segmente. Pigmentlos und blind. Antennen lang und dünn, 5. Antennenglied mehr als 5 mal länger als seine Maximalbreite. Prozonite mit feiner Netzstruktur, glatte Metazonite mit schwach entwickelten Seitenwülsten. $3+3$ Makrochaeten.

Beine ohne Tarsalpapillen. 1. und 2. Beinpaar mit Borstenkämmen am Tarsus. Beinpaare 3-7 beim ô verdickt und mit Veränderungen. 8. und 9. Beinpaar mit Coxalsäcken. 9. Beinpaar mit je einem Coxalfortsatz.

Die vorderen Gonopoden bilden ein Syngonopodit mit bis zur Hälfte ihrer Länge an das Syncoxit verwachsenen Telopoditen. Vorn gibt es lange Gonocoxite, die mit ihren Spitzen die Telopodite überragen; im basalen Teil sind sie zu einem gemeinsamen schmalen Stamm verwachsen. Hinten weist das Syncoxit 2 paramediane, ovale, große Lappen auf sowie 2 kürzere und schmälere seitliche Lappen.

Das Segment der hinteren Gonopoden weist einen medianen schmalen Sternitfortsatz auf, der apikal nach hinten gebogen ist. Die Coxite 
weisen je einen häutigen Coxitfortsatz auf, und die Telopodite erscheinen als gerade mehrgliedrige Stummel.

Generotypus: Dacosoma motasi n.sp.

\section{DACOSOMA MOTASI n. sp.} mitte.

ठึ $14 \mathrm{~mm}$ lang, 1,4 mm breit - 6. Segment, 1,3 mm in der Körper-

o $14 \mathrm{~mm}$ lang, $1 \mathrm{~mm}$ breit in der Körpermitte.

Körper mit 30 Segmenten, vollkommen pigmentlos. Pigmentierte Ocellen fehlen. Kopfkapsel mit dichten und langen Borsten versehen. Stirn leicht gewölbt. Antennen auffällig lang und dünn, ebenfalls mit dichten und langen Borsten versehen (Taf. 1A). 5. Antennenglied 5,36,2 mal länger als seine Maximalbreite. Rand des Labrums auf jeder Seite der 3 gewöhnlichen Zähne abgerundet, ohne Einkerbungen.

Prozonite mit feiner Netzstruktur, Metazonite glatt. Die Seiten. wülste sind schwach entwickelt, auf den hinteren Segmenten verschwinden sie vollkommen. Die Makrochaeten sind lang und befinden sich auf hervorragenden Höckerchen. An den ersten Segmenten sind die Makrochaeten feiner zugespitzt. Der Abstand zwischen den beiden inneren Makrochaeten ist etwa 3mal größer als jener zwischen den inneren Makrochaeten und den mittleren; dieser ist nur etwas größer als der Abstand zwischen den mittleren und äußeren Makrochaeten. Die inneren Makrochaeten befinden sich etwas näher vom hinteren Rand des Segments als die mittleren Makrochaeten. Zwischen der Basis der 3 Makrochaeten befindet sich ein Winkel von ungefähr $100^{\circ}$.

Die Beine weisen keine Tarsalpapillen auf. Das 1. und das 2. Beinpaar sind mit tarsalem Borstenkamm versehen (Taf. 1 D). Die Beinpaare 3-7 sind verdickt und weisen ähnliche Auszeichnungen auf wie die bei Prodicus attemsi Verh. (Strasser 1958, S. 139). Die Beinpaare 3 und 4 sind stark verdickt, und das Präfemur ist am äußeren distalen Teil mit je einem hervortretenden Lappen zur Basis hin versehen (Taf. 2A, B). Die Beinpaare 5-7 sind weniger verdickt, aber stark verlängert. Der Tarsus des 7. Beinpaars (Taf. 2D) ist stark verlängert, so lang wie das Präfemur und das Femur zusammen, und leicht gebogen. Die folgenden Beinpaare sind dünn. 8. und 9. Beinpaare (Taf. $1 \mathrm{E}, \mathrm{F}$ ) mit Coxalsäcken versehen. Die Hüften des 9. Beinpaars weisen hinten, distal innen, je einen zur Basis gebogenen Fortsatz auf. An den folgenden Beinpaaren weisen die Hüften und die Präfemuren in der Mitte des inneren Randes je einen Höcker auf, der immer schwächer entwickelt ist und dann zunächst an den Hüften und später an den Präfemuren verschwindet. 
Die vorderen Gonopoden (Taf. 3 und 4) wie auch bei anderen Antroleucosomiden bilden ein Syngonopodit. Vorn weist das Syncoxit verlängerte blattförmige Gonocoxite auf, voneinander bis nahe zur Basis abgesondert, wo sie zu einem gemeinsamen schmalen Stamm verschmelzen, der von einem trapezoidalen Sockel ausgeht. Im basalen Drittel sind die Gonocoxite auf ihrer Hinterseite an dem zentralen Teil des Syngonopodites verwachsen. Apikal sind die Gonocoxite schräg abgeschnitten und treten an ihrer äußeren Seite als eine Spitze hervor, welche die Telopodite überragt. In der apikalen inneren Ecke sind sie abgerundet und zu einer chitinösen Kante verdickt. Hinten weist das Syncoxit 2 große, ovale, paramediane Lappen auf, die auf ihrer Oberfläche mit vielen Börstchen bedeckt sind. Außer diesen Börstchen weisen die beiden Lappen auch je einen longitudinalen Wulst auf, der mit langen Härchen bedeckt ist. Die beiden Wülste vereinigen sich basal und bilden ein V. Beseitigt man die blattartigen Gonocoxite, so kann man an der Vorderseite dieser Lappen je eine longitudinale Einkerbung sehen, die sich allmählich verliert (Taf. $4 \mathrm{C}$ ). Hinten und seitwärts der beiden paramedianen Syncoxallappen befinden sich kürzere und schmälere Lappen, die an ihrer Oberfläche ebenfalls mit Börstchen versehen sind.

Die Telopodite sind bis zur Hälfte mit dem Syncoxit verwachsen. Im freien, distalen Teil sind sie gebogen und umgeben die blattförmigen Gonocoxite von hinten nach vorn, wobei sie allmählich schmäler werden und in einer Spitze enden.

Das Segment der hinteren Gonopoden weist einen schmalen, apikal zugespitzten und nach hinten gebogenen medianen Sternitfortsatz auf. $\mathrm{Zu}$ beiden Seiten derselben befindet sich je ein häutiger Coxitfortsatz. Die Telopodite erscheinen als mehrgliedrige, gerade, distal gerichtete Stummel. Die Grenze zwischen Coxit und Präfemur ist schwach markiert; etwas ausgeprägter erscheint die Grenze zwischen Präfemur und Femur. Die Tibia ist vom Femur deutlich abgesondert und ist wesentlich schmäler und kürzer als dieser. Das letzte Glied, der Tarsus, ist noch schmäler, sphärisch. Manchmal ist auch eine rudimentere Klaue zu sehen. Im Inneren der Glieder der Telopoditen befindet sich Reduktionspigment.

Beziehungen: Sowohl durch die allgemeine Struktur der vorderen Gonopoden als auch durch das Vorhandensein eines medianen Sternitfortsatzes an dem Segment der hinteren Gonopoden steht vorliegende Art den Arten der Gattung Antroleucosoma nahe. Zwischen den beiden z.Z. bekannten Antroleucosoma-Arten einerseits und Dacosoma motasi andererseits gibt es jedoch glatte Strukturunterschiede der Gonopoden, was die Beschreibung der neuen Gattung rechtfertigt. 
So sind bei den vorderen Gonopoden der beiden AntroleucosomaArten die blattförmigen Gonocoxite untereinander bis zur Basis abgesondert und sind kürzer als die Telopodite, während bei D. motasi die Gonocoxite im basalen Teil zu einem gemeinsamen schmalen Stamm verschmolzen sind und die Länge der Telopodite überschreiten. Äußerst charakteristisch für die Arten der Gattung Antroleucosoma ist das Vorhandensein von 2 hinteren paramedianen Formationen an den vorderen Gonopoden, für die wir die Benennung pseudoflagelloide Fortsätze benützen. Diese Fortsätze fehlen bei den Gonopoden der Art Dacosoma motasi gänzlich oder sind nur durch je eine Borste oder ein Höckerchen vertreten. Desgleichen sind bei den Antroleucosoma-Arten die hinteren Coxallappen (seien sie nun zu einem discoidalen einheitlichen Stück verwachsen wie bei A. spelaea oder deutlich voneinander getrennt wie bei A. banaticum) mit einer gefransten Lamelle bedeckt. Bei Dacosoma motasi sind die hinteren Coxallappen deutlich zu sehen und unbedeckt; die lamellaren Bedeckungsformationen der Antroleucosoma-Arten sind mit den hinteren seitlichen Lappen von Dacosoma motasi wahrscheinlich homolog.

Die hinteren Gonopoden der beiden Antroleucosoma-Arten haben ebenfalls eine einheitliche, gattungscharakteristische Struktur, die von Dacosoma motasi deutlich verschieden ist. So weist das hintere Gonopodensegment bei den Antroleucosoma-Arten einen breiten, medianen Sternitfortsatz auf, mit dem die Coxitfortsätze verschmolzen sind, und die Telopodite erscheinen als winzige Rudimente, die sich seitlich auf einem Femoroid befinden. Bei Dacosoma motasi ist der mediane Sternitfortsatz schmal; es gibt deutliche Coxitfortsätze, und die Telopodite erscheinen als mehrgliedrige, gerade, distal gerichtete Stummel. Das Vorhandensein bei dieser Art von weniger reduzierten Telopoditen als bei allen anderen Antroleucomiden ist natürlich ein primitives Merkmal.

Vorkommen und Material: Höhle ,Peştera Arnăuților", 1 ô (18. VII. 1962, leg. Dan Dancău), 1 (9. VII. 1966, leg. I. Tabacaru); Höhle Nr. 1 in Comarnici-Schlucht, 1 ơ (12. VII. 1966, leg. Doina Dan); Höhle Nr. 2 in Comarnici-Schlucht, 4 ôึ, 6 우, 11 Larven (11. VII. 1966, leg. D. Dancău, Doina Dan und V. Decu); Höhle Nr. 3 in Comarnici-Schlucht, 6 ỗ, 1 q, 4 Larven (13. VII. 1966, leg. I. Tabacaru).

Auf dem südlichen Abhang der Südkarpaten, dem ersten Höhlengebiet westlich von Olt-Tal, befindet sich der Höhenzug BistrițaBuila-Vînturarița-Stogu aus Jura-Kalksteingebilde, der zum CăpățînaGebirge gehört. Im nördöstlichen Teil dieses Kalksteinhöhenzuges, nordwestlich vom Badeort Olăneşti, befinden sich diese Höhlen. Die 
Höhle ,,Peştera Arnăuților" liegt auf dem rechten Ufer des MînzuluiBaches in der Olăneşti-Schlucht (Stogu-Berg) bei etwa 1000 m Höhe und die 3 anderen Höhlen am linken Ufer des Cheia-Baches der Comarnici-Schlucht (Stoguşoarele-Berg) bei etwa 800-900 m Höhe.

\section{Antroleucosoma Verh. 1899}

Der erste bekannte Antroleucosomid, somit die typische Art der Gattung Antroleucosoma Verh., ist A.banaticum Verh. 1899, welche von Verhoeff in der Tatarzy-Höhle im Banat aufgefunden wurde. Diese Höhle, die im Gebiet eigentlich unter dem Namen „,Peştera Mare de la Soronişte“" bekannt ist, liegt am linken Abhang des Cerna-Flusses im Domogled-Berg in der Nähe der Ortschaft Băile Herculane (Herkulesbad). Sie wurde am 14. Juni 1921 von R. Jeannel und E. G. Racoviță erforscht und später, am 8. Juli 1922, von R. Jeannel, bei welcher Gelegenheit ein weiteres of Exemplar der Art Antroleucosoma banaticum Verh. aufgefunden wurde (Biospeologica 1122, Ceuca 1964 a, S. 93). Später wurde diese Höhle des öfteren von den Forschern des Bukarester Institutes für Speläologie erforscht, wobei sowohl 우 als auch $\widehat{o} \widehat{o}$ der Art $A$. banaticum wiedergefunden wurden.

Die zweite Art dieser Gattung, A. (Heteroantroleucosoma) spelaea Ceuca 1964 wurde anhand eines von P. A. Chappuis am 22. VI. 1929 in der Höhle „Piatra Arsă din Cerníşoara“ (Biospeologica 1350) gesammelten Exemplares beschrieben.

Die Überprüfung des Materials von A. banaticum Verh., am typischen Fundort gesammelt, gestattet es uns, die von Verhoeff (1899) angegebene Beschreibung zu ergänzen und auf der Grundlage eines Vergleiches mit der von Tr. Ceuca (1964 a) für die Art A. (Heteroantroleucosoma) spelaea gegebenen Beschreibung die Merkmale der Gattung Antroleucosoma hervorzuheben.

Zum Unterschied zu allen anderen Vertretern der Familie Antroleucosomidae sind die 3-7 Beinpaare des Männchens der Art A. banaticum Verh. dünn und weisen keine besondere Veränderungen auf, so daß Verhoeff dieses Merkmal für die Bestimmung der Gattung Antroleucosoma verwendete (1900, S. 372; 1932, S. 1484). Die Auffindung der zweiten Art, A. (H.) spelaea Ceuca, die durch die Struktur der Gonopoden über ihre Gattungszugehörigkeit überhaupt keine Zweifel aufkommen läßt, die jedoch an den 3-7 Beinpaaren Verdickungen und starke Veränderungen aufweist, beweist, daß der Bau dieser Beine keine Gattungsmerkmale darstellt. Hingegen ergibt ein Studium der Struktur der vorderen und hinteren Gonopoden, die bei den beiden Arten sehr ähnlich sind, eine Reihe von Merkmalen, die für die Gattung Antroleucosoma typisch sind.

Es muß noch hervorgehoben werden, daß sowohl Verhoeff (1899, S. 137) in seiner Beschreibung der Art A. banaticum als auch Ceuca (1964 a, S. 93) in der Beschreibung der Art A.(H.) spelaea Coxitfortsätze am 8. Beinpaar bemerken. Die Erforschung der Art A.banaticum 
gestattete es uns, festzustellen, daß das 9. und nicht das 8. Beinpaar Coxitfortsätze aufweist. Im übrigen ist Verhoeff (1900, S. 395) der gleiche Irrtum bei der ursprünglichen Beschreibung der Art Prodicus attemsi unterlaufen, der nachträglich richtig gestellt wurde (Verhoeff, 1925, S. 91). Was dieses Merkmal anbelangt, möchten wir erwähnen, daß alle Vertreter der Familie Antroleucosomidae Coxitfortsätze am 9. Beinpaar aufweisen; bei einigen jedoch sind es 2 Fortsätze an jedem Coxit, bei anderen nur je 1 Fortsatz.

Unter Beachtung der Merkmale der beiden bekannten Arten können wir für die Gattung Antroleucosoma folgende Diagnose aufstellen:

30 Segmente. Körper vollkommen pigmentlos. Ocellen fehlen. 1. und 2. Beinpaar am Tarsus mit Borstenkamm. Die Beinpaare 3-7 der $\widehat{\jmath}$ sind dünn oder verdickt und mit Veränderungen. Die Beinpaare 8 und 9 mit Coxalsäcken; 9. Beinpaar mit je einem Coxalfortsatz. Die vorderen Gonopoden bilden ein Syngonopodit mit durch eine Lamelle an den zentralen Teil des Syncoxites bis zur Hälfte ihrer Länge verwachsenen Telopoditen. Die Gonocoxiten erscheinen als 2 bis zur Basis voneinander getrennte blattartige Formationen, die am Ende zugespitzt und kürzer sind als die Telopodite. Hinter diesen blattartigen Gonocoxiten gibt es 2 nahe beieinanderliegende ovale Lappen oder ein einheitliches medianes Stück als Ergebnis ihrer Verwachsung. Diese Lappen sind hinten von je einer apikal gefransten Lamelle bedeckt. Ebenfalls an der hinteren Seite gehen von der Basis des Syncoxites 2 paramediane pseudoflagelloide Fortsätze aus. Das Segment der hinteren Gonopoden weist einen medianen breiten Sternitfortsatz auf, mit welchen die Coxitfortsätze verwachsen sind. Die Telopoditrudimente sitzen seitlich auf Femoroiden, welche gegen Ende zu verschmälert sind.

Tr. Ceuca (1964, S. 92) stellt für die Art Antroleucosoma spelaea eine gesonderte Untergattung, Heteroantroleucosoma, auf und gibt zur Unterscheidung der beiden Untergattungen folgende 3 Merkmale (Ceuca 1964, S. 92 in rumänischer Sprache):

Antroleucosoma Verh.

- seitliche Expansionen an den Metazoniten fehlt,

- bei den ô sind die 3.-7. Beinpaare dünn und unverändert, - zwischen den Gonocoxiten und den beiden Verbindungsstücken mit den Femoroiden (vordere Gonop.) gibt es keine andere Formation.
Heteroantroleucosoma Ceuca - seitliche Expansion an den Metazoniten ersichtlich, - bei den ô sind die 3.-7. Beinpaare verdickt und verändert, - zwischen den Gonocoxiten und den beiden Verbindungsstücken mit den Femoroiden (vordere Gonop.) gibt es ein einziges discoidales Stück. 
Was das erste Merkmal betrifft, können wir behaupten, daß es sich mehr um einen quantitativen Unterschied handelt, da die Seitenwülste, die bei $A$. (H.) spelaea stark entwickelt sind, tatsächlich auch bei A. banaticum existieren, aber schwach entwickelt.

In bezug auf das dritte Merkmal muß hervorgehoben werden, daß das einzige discoidale Stück, welches von Ceuca bei $A$. (H.) spelaea beschrieben ist, median eine leichte Trennungslinie aufweist, die klar zeigt, daß sie die Verschmelzung von 2 sehr nahe beieinander liegenden Lappen darstellt, die in der gleichen Stellung bei A. banaticum vorhanden sind.

Unserer Meinung nach können die beiden Untergattungen folgendermaßen getrennt werden:

Die 3.-7. Beinpaare sind dünn und unverändert; die Telopodite der vorderen Gonopoden sind kürzer als die Breite des Syngonopodits; hinter den blattartigen Gonocoxiten gibt es 2 paramediane, ovale Lappen .......... ..... Untergattung Antroleucosoma s. str. (A.[A.] banaticum Verh. 1899)

Die 3.-7. Beinpaare sind verdickt und mit Veränderungen; die Telopodite der vorderen Gonopoden sind länger als die Breite des Syngonopodits; hinter den blattartigen Gonocoxiten gibt es einen einzigen discoidalen, medianen Lappen........ Untergattung Heteroantroleucosoma (A. [H.] spelaea

\section{ANTROLEUCOSOMA (ANTROLEUCOSOMA) \\ BANATICUM Verh.}

1899 Anthroleucosoma banaticum Verhoeff, Arch. Naturg., 65 (1): 137-138, Taf. X, Fig. 45-47.

Die vorderen Gonopoden weisen vorn blattartige Gonocoxite auf, welche am Ende zugespitzt und bis zur Basis voneinander getrennt sind (Taf. $6 \mathrm{~A}$ ). Diese blattartigen Gonocoxite weisen am distalen hinteren Teil je eine senkrechte lamellenartige Verlängerung auf, distal gezähnt. Hinter den blattartigen Gonocoxiten, paramedian, befinden sich 2 ovale Läppchen, welche an ihrer Oberfläche mit dicken Borsten versehen sind. Diese beiden Läppchen sind hinten mit je einer dünnen, apikal langgefransten Lamelle bedeckt. Am distalen Teil sind diese beiden Lamellen wie eine Tüte nach innen gebogen und berühren sich an der medianen Linie. Im mittleren Teil begrenzen die Lamellen, da sie nach innen abgerundet ausgehöhlt sind, einen nahezu runden medianen Raum. An den Rändern, die diesen Raum begrenzen, sind die Lamellen fein und dicht behaart (Taf. 6B). 
An der hinteren Seite gehen von der Basis des Syncoxits 2 paramediane pseudoflagelloide Fortsätze aus, die an ihrer Ansatzstelle eingeschnürt, an ihrem proximalen Teil breit sind und sich allmählich nach dem distalen Teil zu verschmälern, indem sie in eine Spitze auslaufen. Von der Seite gesehen (Taf. 6D), erscheinen diese Fortsätze nach vorn gebogen und enden mit 3 Zähnchen, von denen der innere sehr lang ist.

Die Telopodite erscheinen vorn deutlich von den Gonocoxiten getrennt, hinten jedoch sind sie bis zur Hälfte ihrer Länge durch eine Lamelle mit dem zentralen Teil des Syncoxits verschmolzen. Von der Seite aus betrachtet (Taf. 6C), erscheinen die Telopodite im basalen Drittel verschmälert, dann wieder wie eine Palette zum distalen Teil ausgebreitet.

Das hintere Gonopodensegment weist ein Podosternit (Taf. $5 \mathrm{~A}$ ) mit einem breiten unpaaren Mittelfortsatz auf, der sich distal plötzlich verschmälert und eine dünne mediane Lamelle bildet. Auf beiden Seiten dieser medianen distalen Lamelle gibt es je einen Lappen in Form eines Blattes. Betrachtet man die hintere Seite (Taf. 5 C), so kann man eine mediane Naht der beiden Coxitfortsätze beobachten, die an den hinteren Gonopoden anderer Antroleucosomiden-Gattungen vorhanden und die hier mit dem medianen Sternitfortsatz verschmolzen sind. Die Femoroiden enden in einem schmalen Fortsatz mit unregelmäßig gezahnten Rändern. Seitlich tragen die Femoroiden Telopoditrudimente, die veränderlich sind, 1- oder 2gliedrig, lang wie die Femoroidenfortsätze oder wesentlich kürzer.

Material: Höhle „Peştera Mare dela Soronişte“, 2 ơ ô, 28. V. 1954, leg. Tr. Orghidan; 6 Larven, 18. V. 1962, leg. D. Dancau und I. Taba-

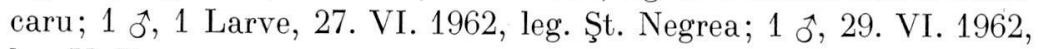
leg. V. Decu.

Bulgarosoma Verh. 1926

Indem Verhoeff (1926a, S. 294; 1926 b, S. 68) die Gattung Bulgarosoma für die Art B. bureschi festlegt, weist er darauf hin, daß sich diese Gattung von der ihr verwandten Gattung Antroleucosoma durch folgende Merkmale unterscheidet:

1. durch eine ausgedehnte mediane Verwachsung des Syncoxits der vorderen Gonopoden, wodurch ein länglicher, kreuzartiger, am Ende ankerartig verbreiterter Aufsatz gebildet wird;

2. durch den Mangel eines mittleren Aufsatzes am Coxosternum des hinteren Gonopodensegments; 
3. durch die Verdickung des 3. und 4. männlichen Beinpaars und verschiedene Auszeichnungen am 3.-7. männlichen Beinpaar;

4. durch eine auffallende Auszeichnung am Vorderkopf des Männchens;

5. durch den matten Rumpf, seine rauhe Skulptur und die stärkeren Seitenwülste desselben.

In Anbetracht der Merkmale der Arten, die später von K. Strasser beschrieben wurden, B. crucis Strass. 1960 und B. rhodopinum Strass. 1966, sowie auch der beiden Arten, die in der Folge beschrieben werden, entspricht diese unterschiedliche Diagnose nicht mehr vollkommen.

Die mediane Verwachsung des Syncoxits der vorderen Gonopoden zu einem großen unpaaren Aufsatz ist für alle Arten der Gattung Bulgarosoma charakteristisch, doch ist dieser Aufsatz nur bei den Arten B. bureschi Verh. und B. crucis Strass. kreuzförmig; bei den anderen Arten weist er keine transversalen Seitenarme auf. Desgleichen muß hervorgehoben werden, daß bei beiden Bulgarosoma-Arten, die in den Höhlen des Rhodopegebirges aufgefunden wurden, B.rhodopinum Strass. und $B$. meridionale n. sp. der unpaare Medianaufsatz keinen ankerartigen Kopf hat.

Die hinteren Gonopoden haben bei allen Bulgarosoma-Arten eine ähnliche Struktur, so daß das Fehlen eines mittleren Aufsatzes am Coxosternum ein gültiges Merkmal für die gesamte Gattung bleibt.

Wie auch bei den anderen Vertretern der Familie Antroleucosomidae (mit Ausnahme der Art Antroleucosoma banaticum) weisen die Arten der Gattung Bulgarosoma an dem 3.-7. Beinpaar der ô Verdickungen und verschiedene Auszeichnungen auf. Diese Veränderungen treten bei $B$. ocellatum n. sp. besonders stark hervor und kommen bei den anderen Arten weniger zum Vorschein.

Im allgemeinen ist der Clipeus bei den ô der Bulgarosoma-Arten flachgedrückt und die Stirn hervorragend, doch ist eine höckerartige Auszeichnung auf dem Vorderkopf ein Merkmal, das nur für die Art B. bureschi Verh. gültig ist.

Was die Skulptur des Teguments und das Gebilde der Seitenwülste anbelangt, so finden wir für die Gattung Bulgarosoma keine typischen Merkmale.

Unter Berücksichtigung der Merkmale der bisher beschriebenen Bulgarosoma-Arten sowie der beiden neuen Arten, B. ocellatum n. sp. und B. meridionale n. sp., kann für diese Gattung folgende Diagnose festgelegt werden:

30 Segmente. Körper pigmentlos. Blind oder mit pigmentierten Ocellen. Antennen lang und schlank; 5. Antennenglied mehr als $3 \mathrm{mal}$ 
länger als seine Maximalbreite. 1. und 2. Beinpaar mit Borstenkamm am Tarsus. 3.-7. Beinpaar des ô verdickt und mit Modifizierungen. 8. und 9. Beinpaar des ô mit Coxalsäcken; 9. Beinpaar mit je einem Coxalfortsatz. Vordere Gonopoden mit einem großen medianen unpaaren Aufsatz, welcher vorn in ein Triangulum erweitert ist. Coxosternum des hinteren Gonopodensegments ohne einen mittleren Aufsatz, sondern mit 2 Paar Fortsätzen, die äußeren länger als die inneren. Die Innenfortsätze sind weit voneinander getrennt.

\section{Bestimmungsschlüssel der Arten der Gattung Bulgarosoma:}

1 (6) Medianaufsatz der vorderen Gonopoden am Ende in einem ankerartigen Kopf verbreitert ....................... 2

2 (5) Medianaufsatz der vorderen Gonopoden kreuzförmig; Triangulum apikal gerade abgeschnitten, nicht in eine mediane Lamelle verlängert; Ocellen fehlen ............................ 3

3 (4) Telopodite der vorderen Gonopoden apikal mit einer tiefgezahnten Randlamelle; Seitenarme des kreuzförmigen Medianaufsatzes lang und nach vorn gebogen; Triangulum mit abgerundetem Proximalwinkel

................................ bureschi Verh.

4 (3) Telopodite der vorderen Gonopoden ohne eine apikale Randlamelle; Seitenarme des kreuzförmigen Medianaufsatzes kurz und sehr wenig gebogen; Triangulum mit zugespitztem Proximalwinkel .......... B. crucis Strass. (B. crucis crucis Strass. und B. crucis lazarevensis

5 (2) Medianaufsatz der vorderen Gonopoden nicht kreuzförmig, da die transversalen Seitenarme fehlen; Triangulum apikal in eine mediane, am Ende zugespitzte Lamelle verlängert; pigmentierte Ocellen vorhanden........................ B. ocellatum n.sp.

6 (1) Medianaufsatz der vorderen Gonopoden am Ende zugespitzt ohne ankerartigen Kopf ....................... 7

7 (8) Triangulum mit zugespitztem Proximalwinkel; pigmentierte Ocellen

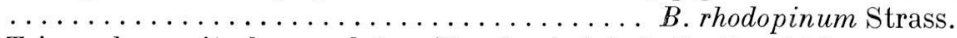

8 (7) Triangulum mit abgerundetem Proximalwinkel; Ocellen fehlen.....

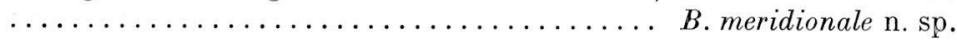

\section{BULGAROSOMA BURESCHI Verh. 1926}

1926 Bulgarosoma bureschi Verhoeff, Zool. Anz., 65: 294-296.

1926 Bulgarosoma bureschi Verhoeff, Arb. Bulg. Naturf. Ges., 12: 69-72,

Taf. III., 1-4.

1926 Bulgarosoma bureschi Verhoeff, Mitt. Bulg. Entom. Ges., 3: 208-210. 1962 Bulgarosoma bureschi, - Strasser, Senck. biol., 43 (6) : 449-450, Abb. 17-19.

Bei den vorderen Gonopoden (Taf. 7) ist der mediane, hintere Aufsatz wie bei B. crucis kreuzförmig, doch sind die Seitenarme hier länger 
und stärker nach vorn gebogen, wobei sie gewöhnlich mit 3 Zähnen enden. Von vorn gesehen, weist das Triangulum ${ }^{2}$ ) einen dieser Art eigenen Aspekt auf (Taf. 8C). So besteht es, wie Verhoeff (1926b) aufgezeigt hat, bei der Art $B$. bureschi aus einem basalen, herzförmigen Teil mit abgerundetem Proximalwinkel und einem trichterförmigen Endteil. Die beiden Teile sind durch eine Verengung voneinander getrennt. Die Seitenränder des Triangulums sind sowohl im verengten als auch im distalen Teil mit langen, nach hinten gebogenen Fransen versehen. Apikal ist das Triangulum gerade abgeschnitten und nur mit vereinzelten Fransen versehen. Von der Seite aus betrachtet (Taf. 8B), kann man die Verbindung zwischen dem hinteren Medianaufsatz und dem Triangulum sehen. An der Basis ist diese Verbindung dick, mit Börstchen bedeckt und mit einem Borstenkamm versehen. Zum distalen Teil zu wird die Verbindung zwischen Aufsatz und Triangulum durch eine dünne, apikal gezahnte Lamelle vertreten, die mit zahlreichen Börstchen und mit kleinen Höckerchen an ihrer Oberfläche versehen ist.

Vorn weist das Syncoxit die von Verhoeff beschriebenen 2 coxalen Kissen auf, die apikal in 2 Hörnchen verlaufen, welche nach außen hin zu beiden Seiten des basalen Teiles des Triangulums gebogen sind. Unter diesen Hörnchen springt das Syncoxit in 2 Lappen hervor, zwischen denen sich eine V-förmige Spaltung bildet, in die das Triangulum hineingepreßt ist. Apikal sind diese beiden Lappen, welche die Seitenarme des hinteren kreuzförmigen Aufsatzes überragen, gerade abgestumpft und mit zahlreichen Härchen bedeckt.

Die äußeren Teile der vorderen Gonopoden, die, wie Verhoeff aufgezeigt hat, als Telopodite betrachtet werden können, kennzeichnen sich bei der Art B. bureschi durch das Vorhandensein einer lamellaren, tiefgezahnten Kante entlang des apikalen Randes mit Ausnahme der inneren Seite. Die apikale Oberfläche der Telopodite ist mit zahlreichen Dörnchen versehen. Vorn erweitern sich die Telopodite zum medianen Teil hin über das Syncoxit zu einer lamellenartigen Formation. Diese Lamelle tritt in ihrem mittleren Teil in Form eines zugespitzten Sporens hervor und verlängert sich distal zu einer Formation, die in 3 Spitzen endet. Hinten weisen die Telopodite zum medianen Teil zu je ein Läppchen auf, das mit Härchen bedeckt ist. Betrachtet man die

2) In der ursprünglichen Beschreibung der Art Bulgarosoma bureschi verwendet Verhoeff für den Teil, auf den wir uns hier beziehen, den Ausdruck ,,Trichteranhang" der Struktur der Gonopoden der Acherosoma mit denen von Bulgarosoma die Benennung Triangulum ein. 
Telopodite von ihrer Innenseite (Taf. 8D), so kann man beobachten, daß sich vom hinteren Lappen aus ein chitinöser, leicht gebogener Arm bildet, der auf seiner Vorderseite mit Börstchen bedeckt ist.

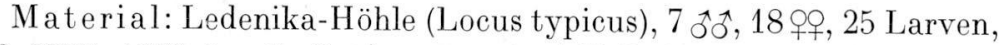
18. VIII. 1963, leg. L. Botoşaneanu und V. Decu.

\section{BULGAROSOMA OCELLATUM n. sp.}

ô 14-14,5 mm lang, 1,5-1,6 mm breit - 6. Segment, 1,3-1,4 mm in der Körpermitte.

o 14-14,5 mm lang, 1,3-1,4 mm breit in der Körpermitte.

Körper mit 30 Segmenten, vollkommen pigmentlos. Beiderseits je 13 pigmentierte Ocellen, in einem Dreieck angelegt. Bei den $\widehat{\jmath}$ ist Clipeus abgeplattet und die Stirn stark gewölbt, ohne jedoch eine höckerartige Auszeichnung wie bei B.bureschi aufzuweisen. Die Antennen sind lang und dünn, schlanker als bei B.bureschi; 5. Glied 4,3-4,8mal länger als seine Maximalbreite.

Der Rand des Labrums ist auf jeder Seite der 3 gewöhnlichen Zähne abgerundet, ohne Einkerbungen.

Die Prozonite mit deutlicher Netzstruktur, körnig erscheinend. Die Metazonite mit schwacher, kaum sichtbarer Netzstruktur. Die Seitenwülste sind abgerundet, an den hinteren Segmenten allmählich schwächer entwickelt. Die Makrochaeten sind kurz und stehen auf kleinen Höckerchen. Auf den ersten und letzten Segmenten sind die Makrochaeten etwas länger und fein zugespitzt. Der Abstand zwischen den inneren Makrochaeten ist doppelt so groß wie der Abstand zwischen den inneren Makrochaeten und den mittleren; dieser wiederum ist etwas größer als der Abstand zwischen den mittleren und den äußeren Makrochaeten. Die inneren Makrochaeten befinden sich auf der gleichen Linie wie die äußeren.

Zwischen der Basis der 3 Makrochaeten bildet sich ein Winkel von ungefähr $115^{\circ}$.

1. und 2. Beinpaar mit dichtem Borstenkamm auf den Tarsen. Das 3.-7. Beinpaar ist bei den Männchen verdickt und weist starke Modifizierungen auf. Beim 3. Beinpaar ist das Präfemur an der Außenseite zu einem abgerundeten Lappen erweitert; das Femur weist auf seiner vorderen Fläche, im basalen Teil, in der Nähe des inneren Randes einen kleinen Höcker auf. Das 4. Beinpaar ist am Präfemur, am äußeren distalen Teil mit einem hervorstehenden Lappen zur Basis hin versehen; der Tarsus ist kurz, birnenförmig, an der Basis verdickt und dem Ende zu stark verschmälert. Das 5. Beinpaar ist am Präfemur wie auch bei den anderen Bulgarosoma-Arten (Strasser 1962, S. 450) 
an der Basis, auf der vorderen Seite mit einem langen, zur Basis hin gebogenen Lappen versehen; der Tarsus ist keulenförmig, stark geschwollen und apikal auf der hinteren Seite zu einem dünnen Lappen verlängert, der über die Kralle hinausragt. Beim 6. Beinpaar ist der Tarsus ebenfalls apikal auf der hinteren Seite zu einem dünnen Lappen verlängert. Der Tarsus des 7. Beinpaars ist stark verlängert, so lang wie das Präfemur und das Femur zusammen, und leicht gebogen.

Die folgenden Beinpaare sind dünn. Das 8. und 9. Beinpaar ist mit Coxalsäcken versehen. Die Coxen des 9. Beinpaars weisen je einen leichtgebogenen Fortsatz auf, und die basale Innenecke bildet einen hervortretenden Winkel.

Beim 11. Beinpaar sind die Coxen und Präfemuren am Innenrand mit je einer Kante versehen, die winkelartig hervortritt. Die Coxen des 11. Beinpaars weisen an der vorderen Oberfläche je einen lamellenförmigen Fortsatz auf, der zur medianen Linie hin als ein abgerundeter Winkel hervortritt, wobei sie sich einander nähern. Am 13. Beinpaar sind die hervortretenden Kanten an der Innenseite der Coxen und des Präfemurs ebenfalls vorhanden, doch erscheint der lamellenförmige Fortsatz an der vorderen Seite der Coxen wesentlich schmäler entwickelt. An den hinteren Beinpaaren der folgenden Segmente (den Beinpaaren 15, 17, 19, 21 usw.) sind all diese hervortretenden Kanten allmählich schwächer entwickelt, bis sie ganz verschwinden, hingegen erscheinen auf der Oberfläche der Coxen und besonders auf der Innenseite zahlreiche kleine kegelförmige Höckerchen.

Die vorderen Gonopoden (Taf. 9 und 10) bilden, wie auch bei den anderen bisher bekannten Arten dieser Gattung, ein Syngonopodit, wobei die Coxiten zu einem medianen, unpaaren, hoch herausragenden Aufsatz gänzlich verschmolzen sind. Von hinten gesehen, erscheint dieser Medianaufsatz an seinem mittleren Teil angeschwollen, weist jedoch keine transversalen Seitenarme auf, wie dies bei den Arten $B$. bureschi und B.crucis der Fall ist, so daß er nicht kreuzförmig ist. Apikal endet dieser Aufsatz in einem ankerartigen Kopf, der jedoch schmäler und länglicher ist als bei den beiden erwähnten Arten, mit zugespitzten Armen, die nach hinten und zur Basis ausgerichtet sind. Nach vorn zu verlängert sich der mediane hintere Aufsatz zu einem Triangulum gleich dem der Art B.crucis mit einem zugespitzten Proximalwinkel und apikal mit 2 schnurrbartförmigen Ästen. Zum Unterschied von B.crucis jedoch sind diese schnurrbartförmigen Äste bei der Art B.ocellatum nicht waagerecht ausgerichtet, sondern distal, schräg. Charakteristisch für die neue Art ist gleichfalls der Umstand, daß sich das Triangulum apikal zu einer medianen Lamelle verlängert, 
die terminal zugespitzt ist und bis zur Höhe des ankerförmigen Kopfes des hinteren Medianaufsatzes gelangt.

Von der Seite aus betrachtet, erscheint der hintere Medianaufsatz (Taf. $10 \mathrm{~B}$ ) stark nach vorn gebogen. Der Verbindungsteil zwischen Medianaufsatz und Triangulum ist mit vielen Börstehen bedeckt und tritt auf beiden Seiten in Form einer winkelförmigen Lamelle hervor. Das terminale Drittel des hinteren Medianaufsatzes ist nach vorn hin zu einer medianen, gezahnten Lamelle verlängert, die an ihrer Oberfläche mit vielen Börstchen bedeckt ist. Diese Lamelle kommt mit dem inneren Rand der medianen apikalen Lamelle des Triangulums in Berührung und schließt auf diese Weise den freien, mittleren Raum.

Vorn weist das Syncoxit eine ähnliche Struktur auf wie das von B.bureschi. Die coxalen Kissen haben die Form eines rechteckigen Dreiecks mit der Spitze zur Basis der Gonopoden ausgerichtet. Zwischen ihnen verbleibt eine mediane Furche, die zur Basis hin breiter wird. Distal sind diese beiden Dreiecke in 2 Hörnchen verlängert, die zu beiden Seiten des medianen Proximalwinkels des Triangulums nach den äußeren Seiten hin gebogen sind. Diese Hörnchen sind länger und schmäler als die von B. bureschi. Hinter den beiden Hörnchen tritt das Syncoxit in Form von 2 median zusammenverwachsenen Lappen hervor, die an ihrem apikalen Teil mit zahlreichen Härchen versehen sind. Diese Lappen, die nur dann zu sehen sind, wenn man die Seitenteile der Gonopoden entfernt, weisen an ihrem hinteren Teil 2 abgerundete Öffnungen auf, deren Rand mit zahlreichen Härchen bedeckt ist. Apikal bieten diese beiden Lappen dem Triangulum einen Halt.

Das Sternit tritt vor den vorderen Gonopoden seitlich in je einem abgerundeten Lappen hervor, der sich zum medianen Teil hin zu je einer Lamelle verlängert. Ein medianer, warziger, 3eckiger Zipfel, wie bei B. bureschi anzutreffen ist, fehlt hier.

Die Seitenteile der Gonopoden, die Telopodite, weisen, von der Seite aus gesehen (Taf. 10A), eine schmale Basis auf und sind distal 2lappig erweitert. Auf ihrer apikalen Oberfläche weisen diese Lappen, besonders der hintere, zahlreiche kleine Dörnchen und Börstchen auf. Betrachtet man die Gonopoden auf ihrer hinteren Seite, so kann man bemerken, daß die hinteren Lappen der Telopodite schmäler sind als die vorderen und median über den Aufsatz gepreßt sind. Vorn sind die Telopodite zum medianen Teil hin über das Syncoxit zu je einer Lamelle verlängert, die sich einander nähern, wobei sie in ihrem mittleren Teil einen ovalen, freien Raum lassen, der zur Basis hin als eine schmale Furche weitergeht. Im distalen Teil weist diese Lamelle am äußeren Rand je eine stark chitinisierte Geschwulst auf, die apikal mit 
langen Fransen versehen ist. Von der Innenseite der Telopodite tritt in der Mitte ein Läppchen hervor, das nach vorn ausgerichtet ist und in zahlreichen langen Stiften endet. Es ist möglich, daß dieses Gebilde eine ähnliche Funktion erfüllt wie ein Grannenapparat.

Die hinteren Gonopoden (Taf. 5 F, G) weisen wie auch bei den anderen Arten dieser Gattung keinen unpaaren medianen Sternitfortsatz auf, sondern 2 Fortsatzpaare - ein inneres und ein äußeres -, wobei das äußere länger ist als das innere.

Die inneren Fortsätze haben eine gemeinsame Basis, an das Sternit anschließende Basis. Sie sind durch eine abgerundete Einbuchtung voneinander abgesondert und gerade oder etwas schräg zur medianen Linie ausgerichtet, wobei sie einander näherkommen. In der distalen Hälfte sind die inneren Fortsätze geschwollen und an ihrer Oberfläche mit zahlreichen Börstchen versehen. Apikal sind diese gerade abgeschnitten, und man kann bemerken, daß sich hier je ein medianes Röhrchen öffnet.

Die stark chitinisierten äußeren Fortsätze, die um ungefähr ein Drittel länger sind als die inneren, sind leierförmig gebogen. Sie verlaufen hinter dem Sockel der inneren Fortsätze und werden von der Basis zum distalen Ende allmählich dünner. Im terminalen Teil sind sie jedoch als Folge des Vorhandenseins von mehreren warzenartigen Erhebungen leicht verbreitert.

Untersuchen wir die hinteren Gonopoden, bevor wir sie von den vorderen loslösen, dann können wir feststellen, daß die äußeren Fortsätze die vorderen Gonopoden seitlich umgeben und die inneren Fortsätze versteckt sind, da sie unter den hinteren Lappen der Telopodite der vorderen Gonopoden eingefügt sind.

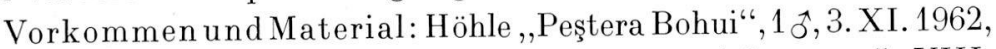
leg. I. Tabacaru; $18 \hat{\jmath} \widehat{\delta}$, zahlreiche Weibchen und Larven, 5. VIII. 1961, leg. A. und Şt. Negrea; 10 ô̋ૈ, zahlreiche Weibchen und Larven, 28. IX. 1961, leg. A. und Şt. Negrea; Höhle „Peştera Ponor-Plopa“,

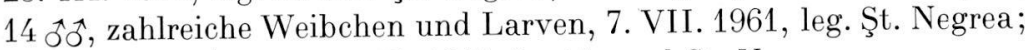
$10 \widehat{\jmath}, 1$ \&, 1 Larve, 26. IX. 1961, leg. A. und Şt. Negrea.

Im Banatergebirge zwischen der Ortschaft Reşița im Norden und der Donau im Süden erstreckt sich ein mesozoisches Kalksteingebiet. Beide obengenannten Höhlen liegen in diesem Gebiet; die Höhle „Peştera Bohui", nördlich der Ortschaft Anina am Bohui-Bach, eine der Quellen des Caraşul-Flusses, und die Höhle ,Peştera Ponor-Plopa südlich von Anina, im Miniş-Becken. In zahlreichen anderen Höhlen des Kalksteingebietes Reşița-Donau wurden Weibchen und Larven der Bulgarosoma gesammelt. Ohne männliche Exemplare kann die Art jedoch nicht bestimmt werden. 


\section{BULGAROSOMA MERIDIONALE n. sp.}

ô $10 \mathrm{~mm}$ lang, 1,1 mm breit - 6. Segment, $1 \mathrm{~mm}$ in der Körpermitte. $+10,5 \mathrm{~mm}$ lang, $1 \mathrm{~mm}$ breit in der Körpermitte.

Körper mit 30 Segmenten, vollkommen pigmentlos. Ocellen fehlen. Bei den ơ ist der Clipeus leicht ausgehöhlt, und die Stirn tritt winklig hervor, ohne jedoch eine höckerartige Auszeichnung aufzuweisen, wie dies bei B.bureschi der Fall ist. Die Antennen sind verhältnismäßig dünn; das 5. Glied ist 3,2-3,6mal länger als seine Maximalbreite.

Der Rand des Labrums ist auf jeder Seite der 3 gewöhnlichen Zähne abgerundet, ohne Einkerbungen.

Die Prozonite und der vordere Teil der Metazonite mit einer feinen Netzstruktur; der Rest der Metazonitenoberfläche ist glatt. Die Seitenwülste sind abgerundet, schwach entwickelt. Die Makrochaeten sind kurz, nur an den ersten Segmenten sind sie etwas länger und zugespitzt. Der Abstand zwischen den inneren Makrochaeten ist ungefähr $1 \frac{1}{2}$ mal größer als jener zwischen den inneren und den mittleren Makrochaeten, und dieser wiederum ist ungefähr 11/2mal größer als der Abstand zwischen den mittleren und den äußeren Makrochaeten. Zwischen der Basis der 3 Makrochaeten befindet sich ein Winkel von etwa $135^{\circ}$.

Das 1. und 2. Beinpaar weist wie auch bei den anderen Antroleucosomiden am Tarsus Borstenkämme auf. Das 3.-7. Beinpaar ist bei den Männchen verdickt, ohne jedoch ausgeprägte Veränderungen aufzuweisen, wie sie bei B.ocellatum anzutreffen sind. Sie sind ähnlich gebildet wie die von B. bureschi. Das Präfemur des 3. und 4. Beinpaars weist auf der Außenseite einen abgerundeten Vorsprung auf. Beim 3. Beinpaar weist der Tarsus auf seiner Innenseite in der Nähe der Basis einen kleinen Vorsprung auf. Am 5. Beinpaar ist wie auch bei den anderen Bulgarosoma-Arten das Präfemur auf seiner Innenseite in der Nähe der Basis mit einem schmalen, länglichen Lappen versehen. An dem 6. und 7. Beinpaar weist das Präfemur an seiner Außenseite einen schwachen Vorsprung auf. Am 7. Beinpaar hat das Femur auf seiner Innenseite keinen mittleren Zapfen, wie dies bei B. bureschi der Fall ist, sondern hat bloß am Ende eine Erweiterung. Tarsus des 7. Beinpaars ist stark verlängert und leicht gebogen.

Das 8. und 9. Beinpaar weist Coxalsäcke auf. Die Coxen des 9. Beinpaars weisen je einen Coxalfortsatz auf, und ihr basaler Innenteil ist mit Tuberkeln bedeckt.

Am Syngonopodit der vorderen Gonopoden ist wie auch bei den anderen Arten der Gattung Bulgarosoma ein unpaarer hochragender Medianaufsatz ausgebildet. Auf ungefähr einem Drittel seiner Länge 
hat dieser Medianaufsatz 2 schräge, an den Rändern gefranste und mit vielen Börstchen bedeckte Seitenarme, und median weist er eine lamellenartige Verlängerung auf. Von der Seite aus gesehen (Taf. 12 B), erscheint diese Lamelle nach vorn gebogen und am Ende wie eine Sichel zugespitzt; an der Vorderseite ist sie mit einem kräftigen und spitzen Zahn versehen. Nach vorn hin wird der Medianaufsatz wie auch bei den anderen Arten der Gattung Bulgarosoma durch ein Triangulum fortgesetzt. Bei vorliegender Art ist das Triangulum stark verlängert und hat einen abgerundeten Proximalwinkel (Taf. 12 C). Apikal hat das Triangulum 2 hörnchenähnliche Arme, die mit langen Borsten bedeckt sind. Median weist das Triangulum einen longitudinalen Wulst auf, der von langen Borsten bedeckt ist und der die apikale Linie überragt. Das Triangulum wird nahezu auf seiner ganzen Oberfläche von Börstchen bedeckt.

Vorn weist das Syncoxit eine ähnliche Struktur auf wie die von $B$. bureschi und B.ocellatum. Wir möchten jedoch erwähnen, daß sich die beiden coxalen Kissen apikal nicht in 2 Hörnchen verlängern, sondern daß nur die beiden Lappen vorhanden sind, die an ihrem Hinterteil je eine längliche Öffnung aufweisen, deren Ränder mit vielen Börstchen versehen sind.

Die Seitenteile der Gonopoden, die Telopoditen, erscheinen, von der Seite aus gesehen, deutlich in 3 Lappen geteilt (Taf. $12 \mathrm{~A}$ ). Der vordere Lappen ist lang, terminal zum medianen Teil gebogen und mit langen Borsten bedeckt. Der hintere Lappen ist apikal abgerundet und mit vielen Wärzchen und Börstchen bedeckt. Der mediane Lappen ist kürzer und ebenfalls apikal abgerundet und von dichten Börstchen bedeckt. Betrachtet man die Vorderseite der Gonopoden (Taf. $11 \mathrm{~A}$ ), so kann man sehen, daß sich die vorderen Lappen der Telopoditen apikal verschmälern und zur medianen Linie hin biegen, wobei sie sich einander nähern. In ihrem mittleren Teil treten die Telopodite mit je einer stark chitinisierten Spitze hervor, und in ihrer basalen Hälfte verlängern sie sich zur medianen Linie hin über das Syncoxit in je eine dünne Lamelle. Diese Lamellen ragen apikal über das Triangulum in je eine Spitze, die am Rande mit Borsten versehen sind.

Betrachtet man die Telopodite auf ihrer Innenseite (Taf. 12 D), so erscheint in der Mitte eine dünne, am Ende zugespitzte, nach vorn gebogene Lamelle, die an ihrem hinteren Rand mit einem langen Sporen versehen ist. Der hintere Lappen wird auf seiner Innenseite im distalen vorderen Teil von langen Zähnen bedeckt, die nach vorn ausgerichtet sind.

Das Sternit der vorderen Gonopoden hat vorn eine ähnliche Struktur wie das von B.ocellatum. Bei der Art B.meridionale gibt es jedoch 
ein medianes Feld, von Wärzchen bedeckt, das aber nicht in einem 3eckigen Zipfel hervortritt, wie dies bei B.bureschi der Fall ist.

Die hinteren Gonopoden (Taf. $5 \mathrm{H}$ ) sind denen von B. ocellatum ähnlich. Die äußeren Fortsätze sind jedoch bei vorliegender Art 2mal länger als die inneren, leicht gebogen, am Ende breiter und mit mehreren Zähnen versehen.

Vorkommen und Material: Höhle ,Imamova dupka“ im Rhodopegebirge, Südbulgarien, 4 ổ, 5 우, 23 Larven, 27. VI. 1966, leg. Tr. Orghidan und Anca Burghele-Balacescu.

\section{Zoogeographische Betrachtungen}

Wie wir in einer früheren Mitteilung aufgezeigt haben ${ }^{3}$ ), gehören die bedeutendsten, echt troglobionten Diplopoden der Balkanhalbinsel und des Karpatengebietes zur Unterordnung Ascospermophora. Aus den Höhlen dieser Gebiete sind Vertreter der Familien Attemsiidae, Scutogonidae, Anthogonidae, Macrochaetosomidae und Antroleucosomidae bekannt.

Verfolgen wir die Verbreitung der Ascospermophoren in den Höhlen der Balkanhalbinsel, dann können wir eine offensichtliche Teilung zwischen dem Osten und dem Westen der Balkanhalbinsel feststellen, wobei als Grenze zwischen diesen Gebieten die Linie Morava-Vardar angesehen werden kann. Im übrigen sind die Karstgebiete des westlichen Teils der Balkanhalbinsel, die sich wie ein breites Band entlang der Julischen Alpen und der ganzen Dalmatischen Küste dahinziehen, von den Karstgebieten des Ostens durch eine ausgedehnte karstfreie Zone getrennt. Diese Trennung der Karstgebiete wie auch ein verschiedener historischer Verlauf der Besiedlung dieser Gebiete bilden die Grundlage der Unterschiede, die wir bei der Verbreitung der troglobionten Ascospermophoren auf der Balkanhalbinsel feststellen. So sind die Familien Attemsiidae, Scutogonidae, Anthogonidae und Macrochaetosomidae in den Höhlen der westlichen Balkanhalbinsel vertreten, während die Familie Antroleucosomidae nur in der östlichen Balkanhalbinsel und den Südkarpaten höhlenbewohnende Vertreter hat.

Die Familie Attemsiidae, die in den östlichen Alpen und im Nordwesten der Dinaren verbreitet ist, hat viele Vertreter, die aus Höhlen bekannt sind, doch handelt es sich nicht um blinde, echte troglobionte

$\left.{ }^{3}\right)$ I. Tabacaru: Einige Bemerkungen über die Verbreitung der Höhlendiplopoden der Balkanhalbinsel und der Karpatengebiete. Mitteilung beim IV. Internationalen Kongress für Speläologie, Postojna-Ljubjana-Dubrovnik, 1965. 
Formen, sondern mehr um troglophile Formen. Die Familien Scutogonidae und Anthogonidae umfassen sowohl höhlenbewohnende als auch an der Oberfläche lebende Arten, die aus dem Westen Europas bekannt sind, und am nordwestlichen Winkel der Balkanhalbinsel ist jede dieser Familien durch je eine endemisch echte troglobionte Gattung vertreten, und zwar die Familie Scutogonidae durch die Gattung Verhoeffeuma mit einer einzigen Art aus einer Höhle vom Triglav (Julische Alpen) und die Familie Anthogonidae durch die Gattung Acherosoma mit zahlreichen Arten, die in den Höhlen der nördlichen Dinaren verbreitet sind. Die Familie Macrochaetosomidae umfaßt nur eine Gattung, Macrochaetosoma, mit höhlenbewohnenden Arten, die in den mittleren und südlichen Dinaren (Bosnien, Herzegowina, Dalmatien) verbreitet sind.

Die östlich der Linie Morava-Vardar bekannten troglobionten Ascospermophoren, und zwar sowohl die aus dem östlichen Teil Serbiens, dem Balkangebirge, Rhodopegebirge, als auch die nördlich der Donau aus dem Banater-Gebirge und den Südkarpaten, gehören ausschließlich zur Familie der Antroleucosomidae.

Laut bisher bekannter Daten haben die Vertreter der Familie Antroleucosomidae folgende Verbreitung:

Gattung Antroleucosoma Verh. 1899

Untergattung ANTROLEUCOSOMA s. str.

A. (A.) banaticum Verh. 1899 - Höhle „,Peştera Mare dela Soronişte“ (= Tatarzy-Höhle) nahe dem Badeort Baile Herculane (Herkulesbad), Banat, Rumänien (Verhoeff 1899, S. 138).

\section{Untergattung HETEROANTROLEUCOSOMA Ceuca 1964}

A. (H.) spelaea Ceuca 1964 - Höhle „Peştera dela Piatra Arsa din Cernişoara“" (Biospeologica 1089), Dorf Sohodol, Gemeinde Pocruia, Oltenien, Rumänien (Ceuca 1964, S. 93).

\section{Gattung Prodicus Attems 1899 \\ Untergattung PRODICUS s. str.}

P. (P.) hispidulus (Silvestri) 1894 mit zahlreichen Unterarten: $P$. (P.) hispidulus hispidulus (Silv.) 1894, P. (P.) hispidulus napolitanus (Attems) 1899, P. (P.) hispidulus appenninorum (Verh.) 1930, P.(P.) hispidulus ischianus Verh. 1940, P. (P.) hispidulus hiemalis Verh. 
1941, P. (P.) hispidulus relictus Verh. 1942, P. (P.) hispidulus quercuum Verh. 1942, P. (P.) hispidulus rotaranus Verh. 1943, P. (P.) hispidulus fissus Verh. 1951, P. (P.) hispidulus sorattinus Verh. 1951, $P .(P$.$) hispidulus epomeanus Verh. 1952$ - Diese Art ist in Italien von Umbria bis Campania einschließlich der Inseln Ischia und Capri verbreitet (siehe C. Strasser 1958, S. 142).

P. (P.) attemsi attemsi Verh. 1900 - Bekannt aus Südfrankreich (nahe Toulouse) und aus Italien von der Toscana und Romagna im Norden bis Monti Albani und Monti Sabini im Süden (siehe C. Strasser 1958, S. 142; J.-P. Mauries 1960, S. 100).

P. (P.) attemsi macchiae (Verh.) 1930 - Oberhalb Porto S. Stefano am Mte. Argentario (Verhoeff 1930, S. 311).

P. (P.) meridionalis (Silv.) 1897 - Sicilia (Messina) (siehe C. Strasser 1958, S. 142).

\section{Untergattung HELLASDICUS Verh. 1940}

P. (H.) penteliconus Verh. 1925 - Pentelikon-Attika, Griechenland (Verhoeff 1925, S. 93).

\section{Untergattung OSMANDICUS Strass. 1960}

P. (O.) bilseli Verh. 1940 - Istambul (Verhoeff 1940, S. 28).

P. (O.) halophilus Verh. 1940 - Nordküste des Marmarameeres nahe von Kücük Cekmece, Türkei (Verhoeff 1940, S. 29).

\section{Untergattung BALKANDICUS Strass. 1960}

P. (B.) albus Strass. 1960 - Höhle ,Pticata dupka“ bei Trojan und Höhle Kumanica bei Cerni Osâm, Bulgarien (Strasser 1960, S. 117; 1962, S. 452).

\section{Gattung Bulgarosoma Verh. 1926}

B. bureschi Verh. 1926 - Höhle Ledenika bei Vraca, Schacht Reznjovete auf den Vracanska planina, Höhle Kitova Kukla beim Dorf Druzevo, Schacht Nevestina propast bei Vraca, Bulgarien (Verhoeff 1926, S. 72; Strasser 1966, S. 344).

B. crucis Strass. 1960 - Höhle „Ravanicka Pecina“ beim Dorf Ravanica am Westfuß der Kucaj-planina östlich von Cuprija und Höhlen „Pecina Vernjikica" und ,Zlotska Pecina" bei Zlot in Ost-Serbien (Strasser 1960, S. 123; 1962, S. 452). 
B. crucis lazarevensis Ceuca 1964 - Höhle ,Lazareva pecina“, nicht weit von Zlot (Biospeologica 956) (Ceuca 1964, S. 41).

B.rhodopinum Strass. 1966 - Höhle Erkjuprija beim Dorf Mostovo im Zentral-Rhodopegebirge, Bulgarien (Strasser 1966, S. 348).

B.ocellatum n. sp. - Höhlen ,Peştera Bohui" und ,Peştera PonorPlopa" in der Umgebung der Stadt Anina, Banat, Rumänien.

B. meridionale n. sp. - Höhle ,Imamova dupka", Rhodopegebirge, Bulgarien.

\section{Gattung Paraprodicus Verh. 1940}

P. penicillatus Attems 1902 - Aselakia-Wald in Askiphu, Kreta (Attems 1902, S. 588).

\section{Gattung Bulgardicus Strass. 1960}

B.tranteevi Strass. 1960 - Höhle „Bankowitza“ bei Karlukowo in NW-Bulgarien (Strasser 1960, S. 112).

\section{Gattung Dacosoma n. g.}

D.motasi n. sp. - Höhle „Peştera Arnauților" und Höhlen Nr. 1, 2 und 3 in der Comarnici-Schlucht, nordwestlich des Badeortes Olaneşti, Rumänien.

Aus obenstehenden Angaben ist zu ersehen, daß die Familie Antroleucosomidae in den Mittelmeergebieten vom Süden Frankreichs bis in den europäischen Teil der Türkei verbreitet ist; der am südlichsten gelegene Punkt ihrer Verbreitung ist die Insel Kreta, und die Nordgrenze befindet sich in den Südkarpaten. Diese Familie umfaßt, wie wir gesehen haben, eine weitverbreitete Gattung, Prodicus, die sich in 4 Untergattungen teilt: Prodicus s. str. mit 3 Arten, die im Süden Frankreichs, der italienischen Halbinsel, den Inseln Ischia, Capri und Sizilien verbreitet sind; Hellasdicus Verh. mit 1 Art in Griechenland; Osmandicus Strass. mit 2 Arten im europäischen Teil der Türkei und Balkandicus Strass. mit 1 Art aus dem mittleren Balkangebirge.

Die anderen Gattungen haben eine wesentlich weniger umfangreiche Verbreitung. Die Gattung Paraprodicus Verh. ist mit 1 Art von der Insel Kreta bekannt und die Gattung Bulgardicus Strass. mit nur 1 Art aus dem mittleren Balkangebirge. Die Gattung Bulgarosoma Verh. mit 5 Arten ist aus dem Rhodopegebirge, dem westlichen Balkangebirge, den Karpaten südlich der Donau (dem Gebiet zwischen Moravatal und Nisava-Timoc-Korridor) und dem Banater-Gebirge bekannt. Die Verbreitung der Gattung Bulgarosoma überschreitet östlich 
nicht den Korridor Timiş-Cerna in den Südkarpaten; hingegen sind für die Karstgebiete der Südkarpaten die Gattungen Antroleucosoma mit 2 Arten und Dacosoma mit 1 Art endemisch.

Die Gattungen Antroleucosoma, Bulgarosoma, Dacosoma, Bulgardicus und die Untergattung Balkandicus (Gattung Prodicus) sind ausschließlich aus Höhlen bekannt. Mit Bestimmtheit troglobionte, blinde Arten sind: Antroleucosoma (A.) banaticum, A. (Heteroantroleucosoma) spelaea, Dacosoma motasi, Bulgarosoma bureschi, B. crucis und B. meridionale. Was die Art Bulgarosoma ocellatum anbelangt, so können wir, trotzdem sie pigmentierte Ocellen aufweist, in Anbetracht der Depigmentierung des Körpers und der Tatsache, daß sie ausschließlich in Höhlen angetroffen wurde, behaupten, daß sie ebenfalls eine troglobionte Art ist. Es ist möglich, daß die Situation bei der Art Prodicus (Balkandicus) albus die gleiche ist.

Somit hat die Familie Antroleucosomidae höhlenbewohnende Vertreter nur in der östlichen Hälfte der Balkanhalbinsel beziehungsweise im Westen Bulgariens (in den westlichen und mittleren Balkangebirgen und im Rhodopegebirge), im östlichen Teil Serbiens (den Karpaten südlich der Donau) und nördlich der Donau im Südwesten Rumäniens (im Banater-Gebirge und den Südkarpaten bis zum Olt-Fluß). Wie wir gezeigt haben, sind in diesen Gebieten die Antroleucosomiden die einzigen troglobionten Vertreter der Unterordnung Ascospermophora. In Anbetracht der Konzentrierung von Strukturtypen ist es möglich, daß das ostbalkanische Gebiet auch das Differenzierungs- und Verbreitungszentrum der Familie Antroleucosomidae gewesen ist.

Die Verbreitung der Antroleucosomiden in den Südkarpaten wie auch die der troglobionten Coleopteren (Jeannel 1931, S. 53; 1943, S. 177) überschreitet das Olt-Tal nicht nach Osten. Wir müssen jedoch bemerken, daß, während andere Gruppen von höhlenbewohnenden Arthropoden (Coleoptera-Batiscinae und Trechinae, Isopoda-Trichoniscidae, Diplopoda-Typhloiulini) auch nördlich des Mureş-Tales in den Höhlen des Bihargebirges vertreten sind, die Verbreitung der Antroleucosomidae die Südkarpaten als nördliche Grenze nicht überschreitet. Somit bilden im Osten Europas die Südkarpaten die nördlichste Grenze der Verbreitung der troglobionten Ascospermophoren.

Wenn wir die Höhlenfauna der Südkarpaten und des BanaterGebirges in ihren phyletisch-zoogeographischen Beziehungen untersuchen, so können wir hervorheben, daß, während die cavernicolen Coleopteren Batiscinae und Trechinae dieser Gebiete (so wie die aus dem Bihargebirge) ihren Ursprung in den mittleren Dinaren haben (Jeannel 1943, S. 172, 203), die cavernicolen Diplopoda Ascospermophora Affinitäten mit denen der Rhodope- und Balkangebirge aufweisen. 


\section{ZUSAMMENFASSUNG}

Die Familie Antroleucosomidae ist im Mittelmeergebiet vom Süden Frankreichs bis in den europäischen Teil der Türkei verbreitet; der am südlichsten gelegene Punkt ihrer Verbreitung ist die Insel Kreta, und die Nordgrenze befindet sich in den Südkarpaten. Aus dieser Familie sind bis jetzt folgende Gattungen bekannt: Antroleucosoma Verh. (mit den Untergattungen Antroleucosoma s. str. und Heteroantroleucosoma Ceuca), Prodicus Attems (mit den Untergattungen Prodicus s. str., Hellasdicus Verh., Osmandicus Strass. und Balkandicus Strass.), Bulgarosoma Verh., Paraprodicus Verh. und Bulgardicus Strass.

In der vorliegenden Arbeit wird eine neue Gattung Dacosoma mit der Art $D$. motasi aus Höhlen von den Südkarpaten beschrieben sowie 2 neue Bulgarosoma-Arten, und zwar B. ocellatum n. sp. aus Höhlen des BanaterGebirges und $B$. meridionale n. sp. aus einer Höhle des Rhodopegebirges. Ebenfalls ist die Beschreibung der Arten Antroleucosoma banaticum Verh. 1899 und Bulgarosoma bureschi Verh. 1926 ergänzt auf Grund der Untersuchung von Material aus Höhlen, aus denen sie ursprünglich beschrieben wurden. Zugleich ist ein Bestimmungsschlüssel für die Gattungen der Familie Antroleucosomidae sowie ein Bestimmungsschlüssel für die Arten der Gattung Bulgarosoma aufgestellt.

Laut der bisher bekannten Daten hat die Familie Antroleucosomidae höhlenbewohnende Vertreter in den Südkarpaten, westlich des Olt-Flusses (Antroleucosoma banaticum, A. [H.] spelaea, Dacosoma motasi). im BanaterGebirge (Bulgarosoma ocellatum), in den Karpaten südlich der Donau beziehungsweise aus dem Gebiet zwischen dem Morava-Tal und dem NisavaTimoc-Korridor (Bulgarosoma crusis), im Balkangebirge (Bulgarosoma bureschi, Bulgardicus tranteesi, Prodicus [Balkandicus] albus) und im Rhodopegebirge (Bulgarosoma rhodopinum und B. meridionale). In diesen Gebieten sind die Antroleucosomiden die einzigen troglobionten Vertreter der Unterordnung Ascospermophora. Es ist zu bemerken, daß die Verbreitung der cavernicolen Antroleucosomiden nach Westen die Linie Morava-Vardar und nach Norden die Südkarpaten nicht überschreitet.

\section{RÉSUMÉ}

La famille des Antroleucosomidae est connue dans la région méditerranéenne; son aire de dispersion s'y étend du Sud de la France à la partie européenne de la Turquie, limitée qu'elle est au Nord par les Carpates méridionales et au Sud par l'île de Crète. Jusqu'à présent cette famille était représentée par les genres suivants: Antroleucosoma Verh. (avec les sousgenres Antroleucosoma s. str. et Heteroantroleucosoma Ceuca), Prodicus Attems (avec les sous-genres Prodicus s. str., Hellasdicus Verh., Osmandicus Strass., et Balkandicus Strass.), Bulgarosoma Verh., Paraprodicus Verh. et Bulgardicus Strass.

Dans ce travail l'auteur décrit un nouveau genre Dacosoma avec l'espèce $D$. motasi n. sp. des grottes des Carpates méridionales ainsi que deux nouvelles espèces de Bulgarosoma, à savoir $B$. ocellatum $\mathrm{n}$. sp. des grottes des Monts du Banat et $B$. meridionalis n. sp. d'une grotte du Mont Rhodope ; de plus il complète la description des espèces Antroleucosoma banaticum 
Verh. 1899 et Bulgarosoma bureschi Verh. 1926 en se basant sur l'étude de matériel provenant des grottes d'où on les avait décrites initialement. L'auteur établit en même temps, une clé de détermination pour les genres de la famille Antroleucosomidae ainsi qu'une clé pour les espèces du genre Bulgarosoma.

Selon ce que l'on connaît jusqu'à présent, la famille Antroleucosomidae possède des représentants cavernicoles dans les Carpates méridionales à l'Ouest de l'Olt (Antroleucosoma banaticum, A. [H.] spelelaea, Dacosoma motasi), dans les Monts du Banat (Bulgarosoma ocellatum), dans les Carpates du Sud du Danube, c'est-à-dire dans la région comprise entre la vallée de la Morava et le couloir Nisava-Timoc (Bulgarosoma crucis), dans les Monts Balkans (Bulgarosoma bureschi, Bulgardicus tranteewi, Prodicus [ Balkandicus] albus) et dans le Mont Rhodope (Bulgarosoma rhodopinum et Bulgarosoma meridionale). Dans ces régions les Antroleucosomides sont les seuls représentants troglobies du sous-ordre Ascospermophora. Il est à remarquer que la dispersion des Antroleucosomides cavernicoles ne dépasse pas à l'Ouest la ligne Morava-Vardar et ni au Nord celle des Carpates méridionales.

\section{LITERATUR}

Aтtems, C. (1899) - Neues über paläarktische Myriopoden. Zool. Jb., Syst. 12: $286-336$.

- (1902) - Myriopoden von Kreta, nebst Beiträgen zur allgemeinen Kenntnis einiger Gattungen. SB. Ak. Wien math.-naturw. CI., CXI, 1: $527-614$

- (1959) - Die Myriopoden der Höhlen der Balkanhalbinsel. Nach dem Material der ,Biospeologica balcanica“. Ann. naturhist. Mus. Wien 63: 281-406.

Ceuca, T. (1964 a) - Cîteva diplopode noi în fauna R.P.R. Studia Universitatis Babeş-Bolyai, Series Biologia 2: 89-99.

- (1964 b) - Zur Kenntnis der Höhlendiplopoden Jugoslawiens. Fragmenta Balcanica Musei Maced. Sci. Nat. V, 8: 37-46.

Dancau, D., und Tabacaru, I. (1964) - Observații zoogeografice asupra faunei cavernicole din Oltenia şi Banat. Lucr. Inst. Speol. ,,Emil Racoviță“ III : 293-340.

JeAnneL, R. (1931) - Origine et évolution de la faune cavernicole du Bihar et des Carpathes du Banat. Atti dell'XI Congresso internazionale di Zoologia, Padova 1930. Arch. Zool. Ital. XVI : 47-60.

- (1943) - Les fossiles vivants des cavernes. : 1-321. Gallimard, Paris.

Latzel, R. (1884) - Die Myriopoden der Österreichisch-Ungarischen Monarchie. Zweite Hälfte: 1-414. Alfred Hölder, Wien.

Mauriès, J.-P. (1960) - Diplopodes de la région toulousaine. Bull. Soc. Hist. nat. Toulouse 95: 100-104.

Strasser, C. (1958) - Diplopodi del Lazio. Boll. Soc. Adriatica Sci. Nat. Trieste XLIX, 9: 137-170.

- (1960) - Diplopoden aus Alpen-, Apenninen- und Balkanländern. Fragmenta Entomologica Roma III, 6: 95-140.

- (1962) - Diplopoden aus Bulgarien und Jugoslawien. Senck. biol. 43, 6: 437-470.

- (1966) - Über Diplopoden Bulgariens, Annales Zoologici, Warszawa XXIII, 12: 325-385. 
Verhoeff, K. W. (1899) - Beiträge zur Kenntnis paläarktischer Myriopoden. VIII. Aufsatz: Zur vergleichenden Morphologie, Phylogenie, Gruppenund Artsystematik der Chordeumiden. Arch. Naturg. 65, 1: 95-154.

- (1900) - Beiträge zur Kenntnis paläarktischer Myriopoden. XIII: Zur vergleichenden Morphologie, Phylogenie, Gruppen- und Artsystematik der Ascospermophora. Arch. Naturg. 66, 1: 347-402.

- (1909) - Neues System der Diplopoda-Ascospermophora. Zool. Anz. XXXIV, 18/19: 566-572.

- (1910) - Über Diplopoden. 11.-15. Aufsatz (31.-35.) : Beiträge zur Kenntnis der Glomeriden, Juliden, Ascospermophoren und Lysiopetaliden sowie zur Fauna Siziliens. Nova Acta Leopoldina 92: 139-448.

- (1914) - Ascospermophoren aus Japan (über Diplopoden, 69. Aufsatz). Zool. Anz. XLIII, 8: 342-370.

- (1925) - Neue Diplopoden-Beiträge. 95. Diplopoden-Aufsatz. Zool. Jb. Syst. 50: 61-122.

- (1926a) - Zwei neue Höhlen-Myriopoden aus Bulgarien. Eine vorläufige Mitteilung. Zool. Anz. 65, 11/12: 294-296.

- $(1926$ b) - Über einige von Dr. I. Buresch in Bulgarien gesammelte Diplopoden. 1. Aufsatz. Arb. Bulgar. Naturf. Ges. 12: 67-78.

- $(1926 \mathrm{c})$ - Über einige von Dr. I. Buresch in Bulgarien gesammelte Diplopoden. 2. Aufsatz. Mitt. Bulgar. Entom. Ges. 3: 193-210.

- (1929) - Arthropoden aus südostalpinen Höhlen, gesammelt von Herrn Karl Strasser, 2. Aufsatz. Mitt. Höhlen- u. Karstforsch. 2: 41-55.

- (1930 a) - Arthropoden aus südostalpinen Höhlen, gesammelt von Karl Strasser, 3. Aufsatz: Acherosoma und seine verwandtschaftlichen Beziehungen. Mitt. Höhlen- u. Karstforsch. 1: 1-12.

- (1930 b) - Zur Kenntnis italienischer Diplopoden. 119. Diplopoden-Aufsatz. Zool. Jb. Syst. $60: 281-326$.

- (1932) - Klasse Diplopoda in Bronns Klassen und Ordnungen des Tierreichs, 5, Abt. II : 1-2084.

- (1940 a) - Über einige Diplopoden, Chilo- und Isopoden der Insel Ischia. Zool. Anz. 131, 11/12: 271-287.

- (1940 b) - Über Diplopoden aus der Türkei. Istanb. Univ. Fen Fakült. Mecmuasi, Ser. B, V, 1/2:1-49.

\section{ERKLÄRUNG DER TAFELN 1 (1)-13 (13)}

\section{TAF EL 1 (1)}

Dacosoma motasi n.g.n.sp. ô (Höhle ,Peştera Arnăutilor"). A - Antenne; $\mathrm{B}-\mathbf{1 5}$. Segment, Dorsalansicht; $\mathrm{G}$ - dasselbe, von hinten gesehen; D - linkes 1. Bein, von vorn gesehen; E - Grundteil des 8. Beinpaars, von hinten gesehen; F - rechte Hälfte des Grundteiles des 9. Beinpaars, von hinten gesehen.

TAFEL $2(2)$

Dacosoma motasi n.g.n.sp. ơ (Höhle „Peştera Arnăuţilor“). A - rechtes 3. Bein, von hinten gesehen; B - rechtes 4. Bein, von hinten gesehen; C linkes 5. Bein, von vorn gesehen; D - linkes 7. Bein, von vorn gesehen. 
TAFEL $3(3)$

Dacosoma motasi n.g.n.sp. đ̊ (Höhle „Peştera Arnăuților“). A - vordere Gonopoden, von vorn gesehen; B - dasselbe, von hinten gesehen.

\section{TAFEL $4(4)$}

Dacosoma motasi n.g.n.sp. ơ (Höhle „Peştera Arnăuților“). A - vordere Gonopoden, Seitenansicht; B - Gonocoxite, Seitenansicht; C - vordere Gonopoden nach Entfernung der Gonocoxite, von vorn gesehen.

\section{TAFEL $5(5)$}

A-C - Antroleucosoma banaticum Verh. ô. A - hintere Gonopoden, von vorn gesehen; B - Endhälfte des Sternitfortsatzes in Seitenansicht; C - dasselbe, von hinten gesehen. D und E - Dacosoma motasi n.g.n.sp. $\sigma^{\wedge}$ (Höhle „Peştera Arnăuților"). D - hintere Gonopoden, von vorn gesehen; E - Endhälfte des Sternitfortsatzes in Seitenansicht. F und G - Bulgarosoma ocellatum n.sp. ơ (Höhle „Peştera Bohui“"). F - hintere Gonopoden, von hinten gesehen; $\mathrm{G}$ - dasselbe, von vorn gesehen. $\mathrm{H}$ - Bulgarosoma meridionale n.sp. ơ, rechte Hälfte der hinteren Gonopoden, von hinten gesehen.

TAFEL 6 (6)

Antroleucosoma banaticum Verh. $\sigma^{t}$. A - vordere Gonopoden, von vorn gesehen; B - dasselbe, von hinten gesehen; C - dasselbe in Seitenansicht; D - linke Hälfte der vorderen Gonopoden, von der Innenseite gesehen.

\section{TAFEL 7 (7)}

Bulgarosoma bureschi Verh. §. A - vordere Gonopoden, von vorn gesehen; $\mathrm{B}$ - dasselbe, von hinten gesehen.

\section{TAFEL 8 (8)}

Bulgarosoma bureschi Verh. ô. A - vordere Gonopoden, Seitenansicht; B - Medianaufsatz der vorderen Gonopoden, Seitenansicht; C - dasselbe, von vorn gesehen; D - linker Telopodit der vorderen Gonopoden, von der Innenseite gesehen.

\section{TAF EL 9 (9)}

Bulgarosoma ocellatum n.sp. ô (Höhle „Peştera Bohui“). A - vordere Gonopoden, von vorn gesehen; B - dasselbe, von hinten gesehen.

\section{TAF E L $10(10)$}

Bulgarosoma ocellatum n.sp. ơ (Höhle „Peştera Bohui“). A - vordere Gonopoden, Seitenansicht; B - Medianaufsatz der vorderen Gonopoden, Seitenansicht; C - dasselbe, von vorn gesehen; D - linker Telopodit der vorderen Gonopoden, von der Innenseite gesehen. 
TAFEL 11 (11)

Bulgarosoma meridionale n.sp. $\sigma^{\star}$. A - vordere Gonopoden, von vorn gesehen; B - dasselbe, von hinten gesehen.

TAFEL 12 (12)

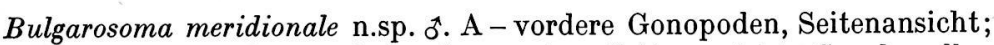
B - Medianaufsatz der vorderen Gonopoden, Seitenansicht; C - dasselbe, von vorn gesehen; D - linker Telopodit der vorderen Gonopoden, von der Innenseite gesehen.

TAF EL 13 (13)

Verbreitungskarte der Familie Antroleucosomidae. 

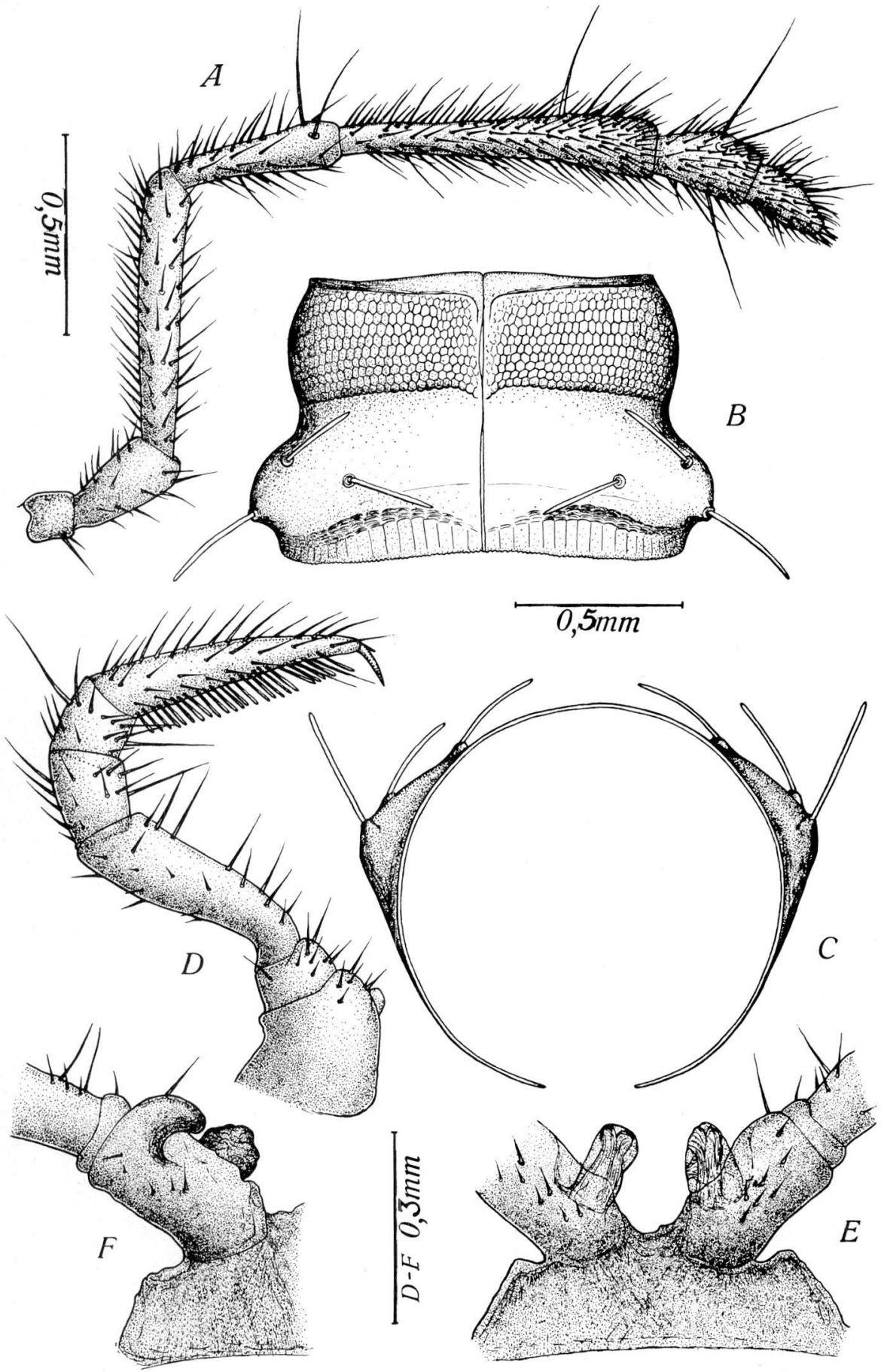


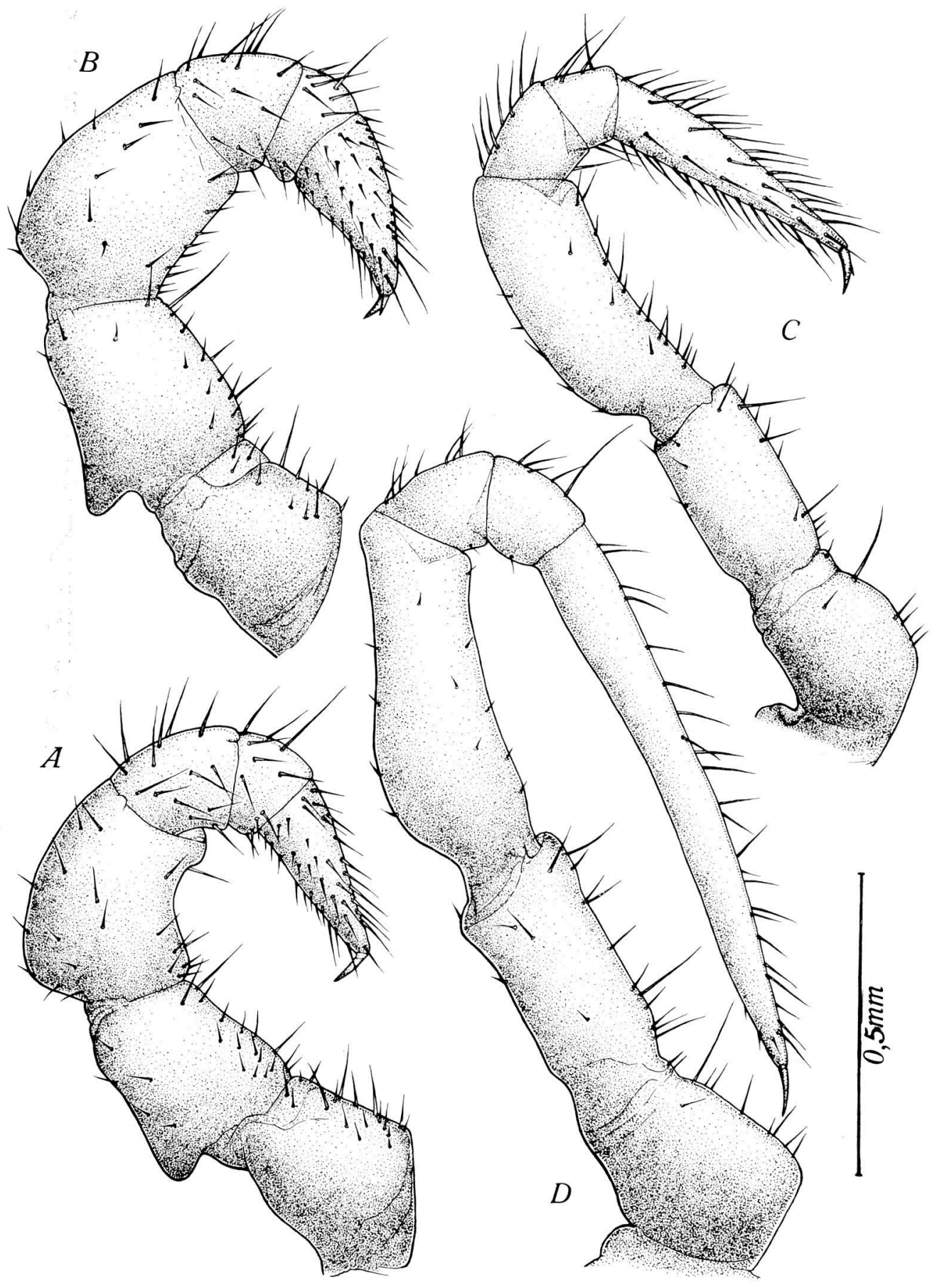



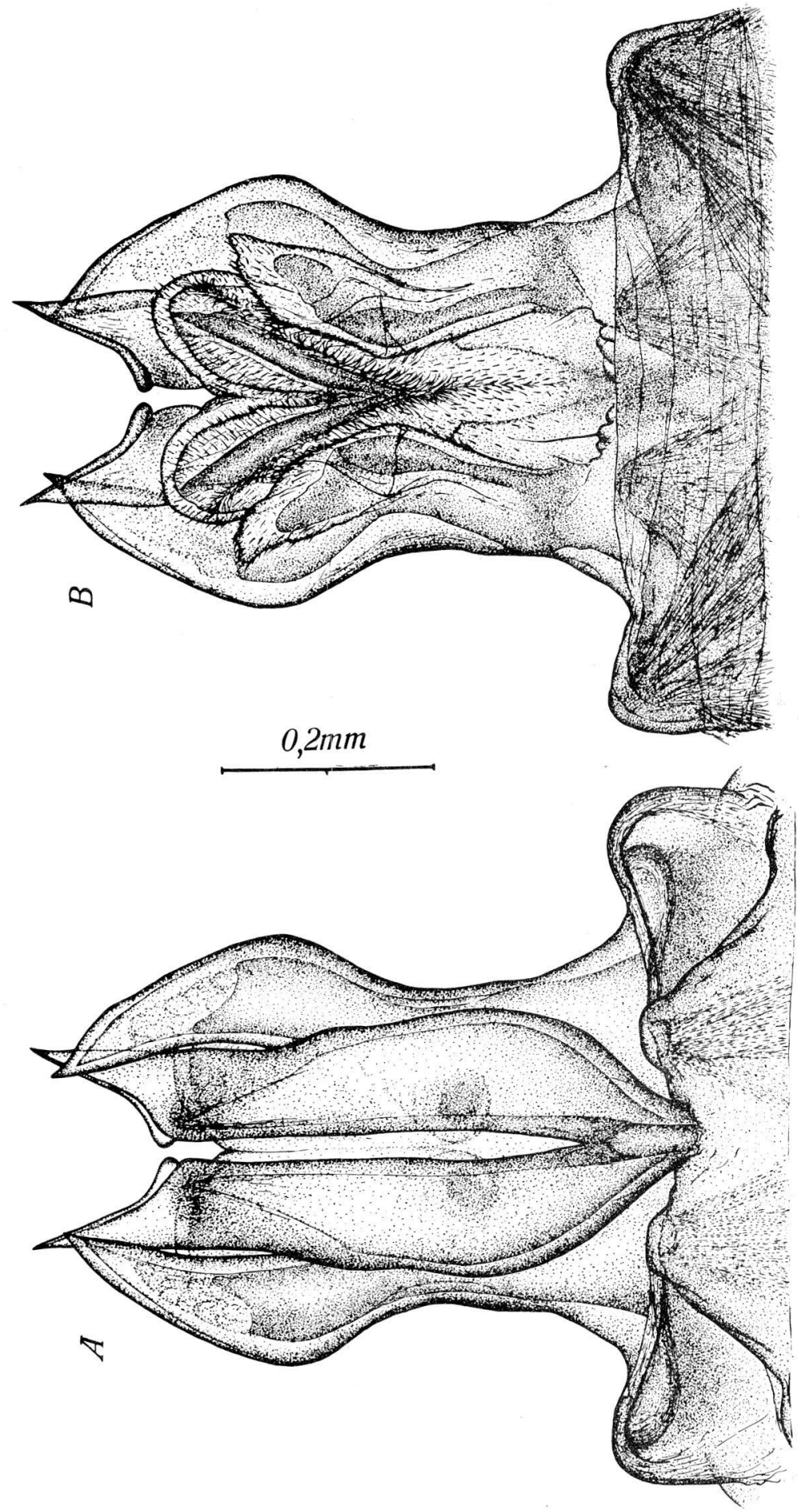

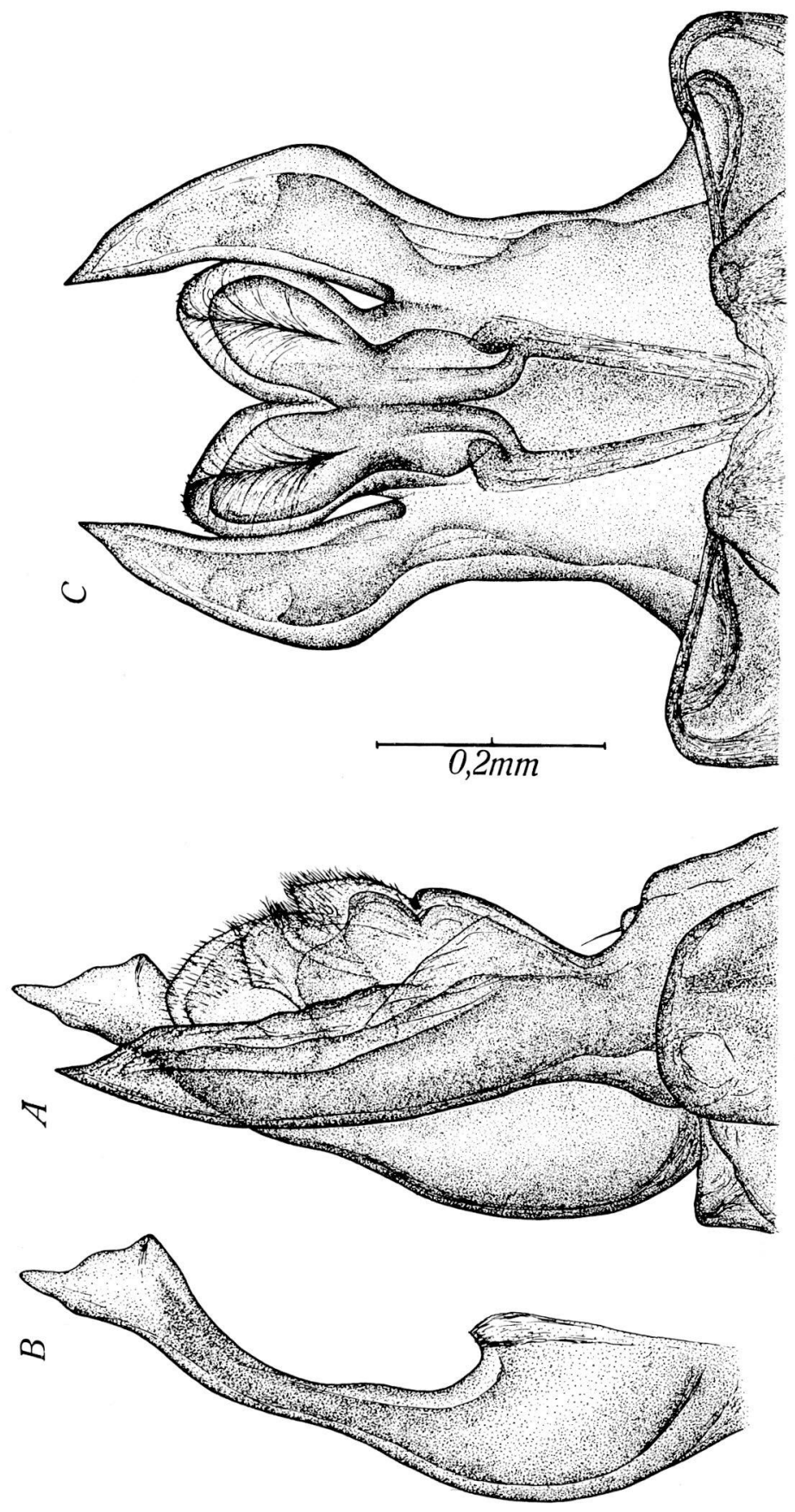


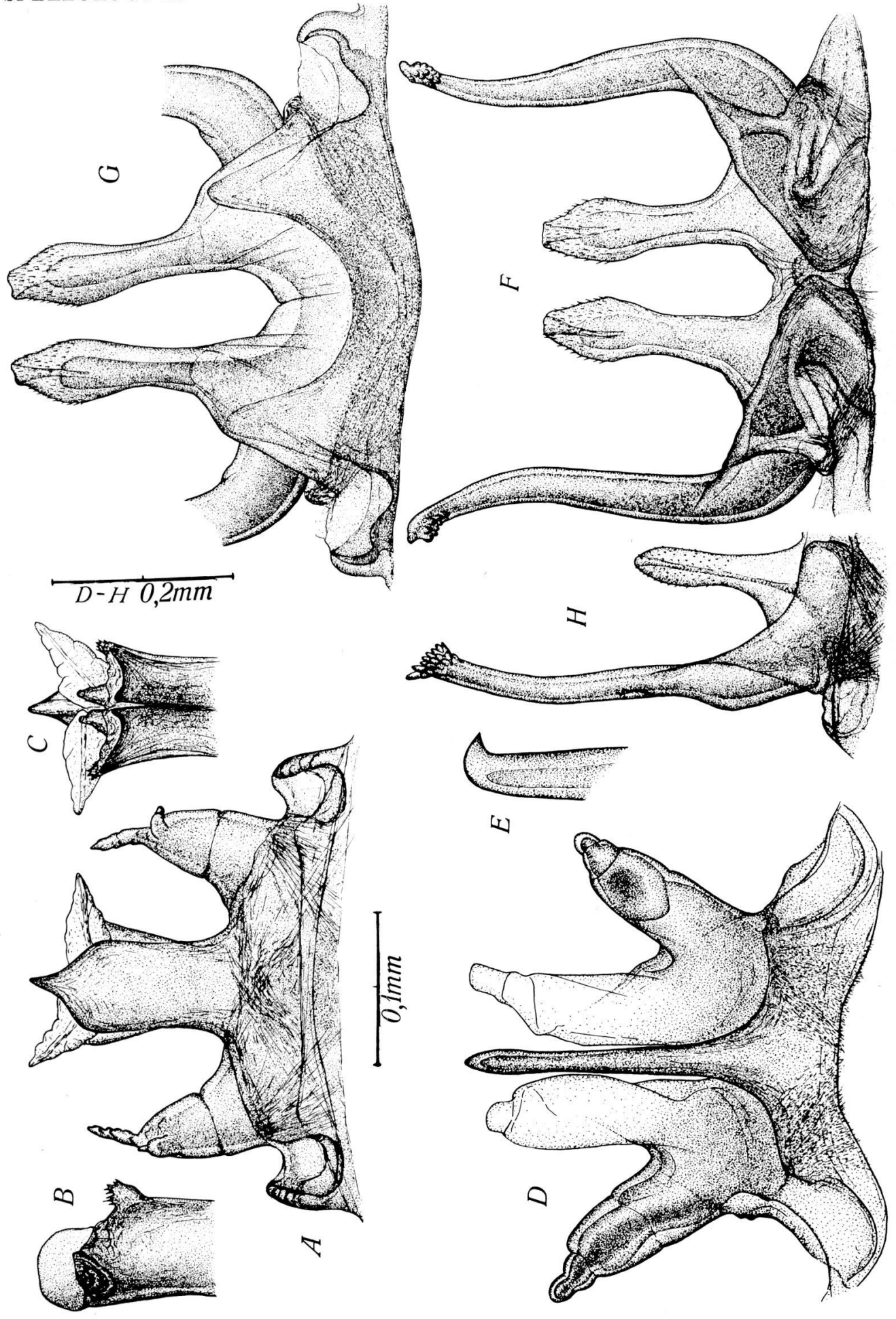



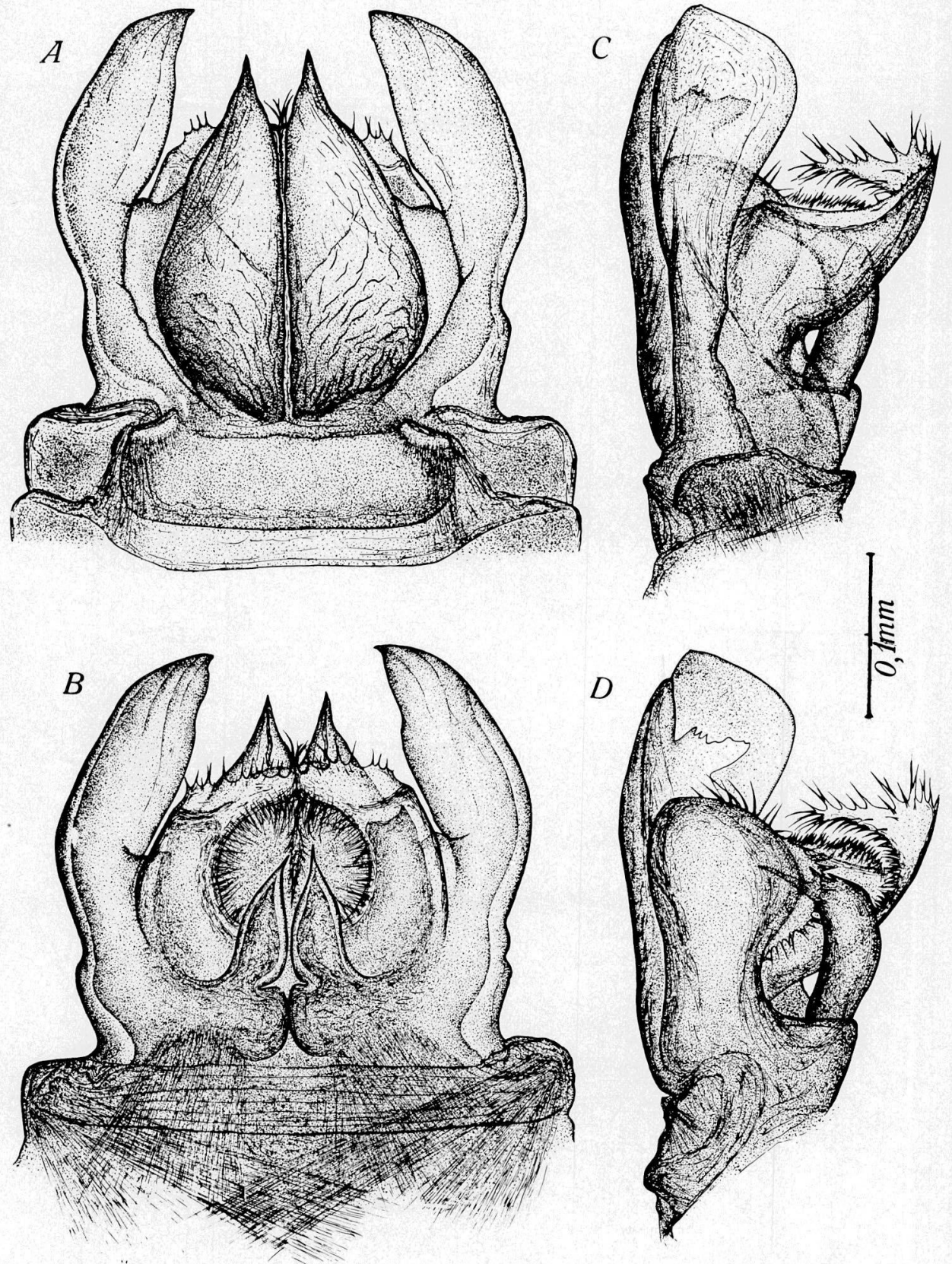

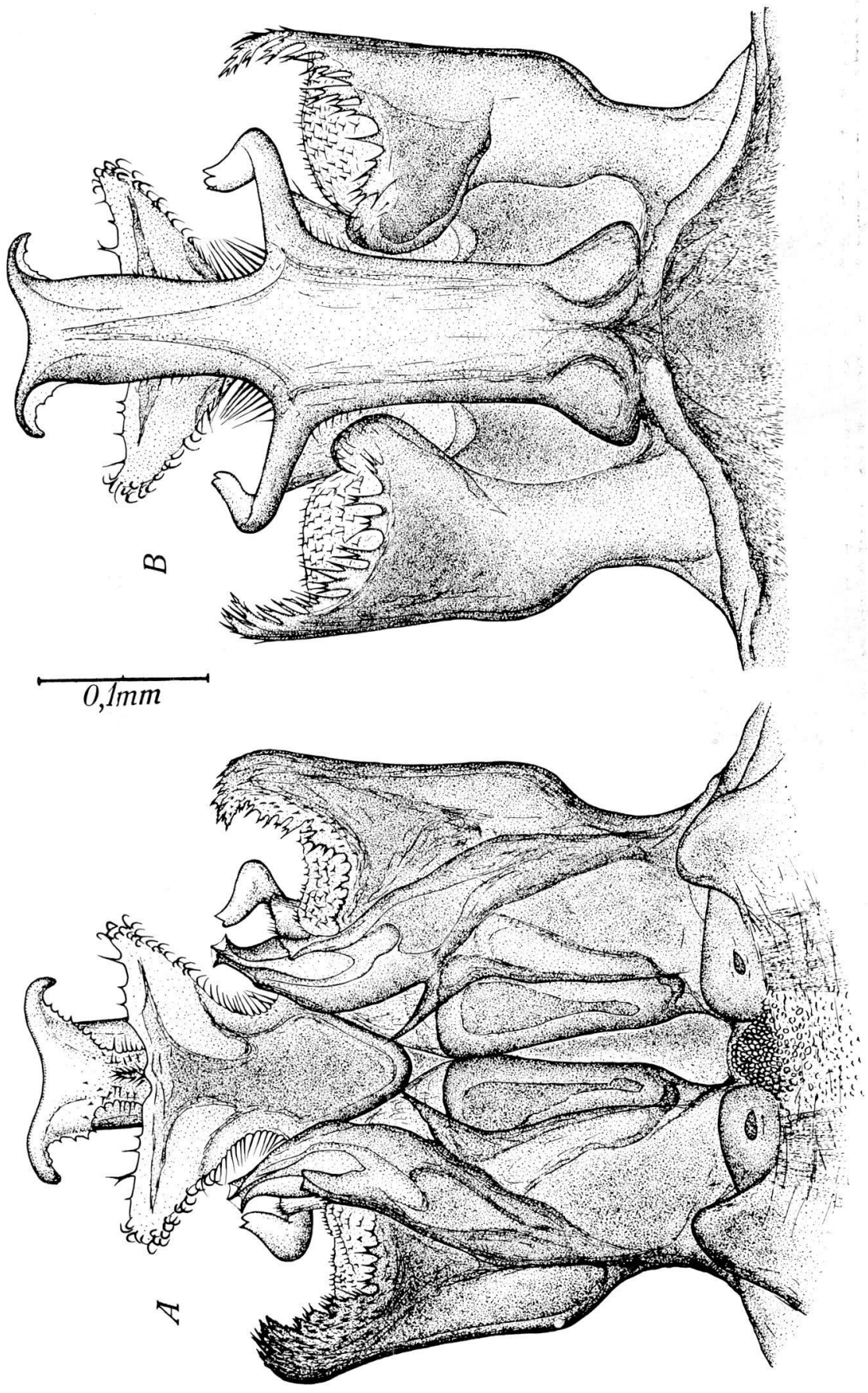

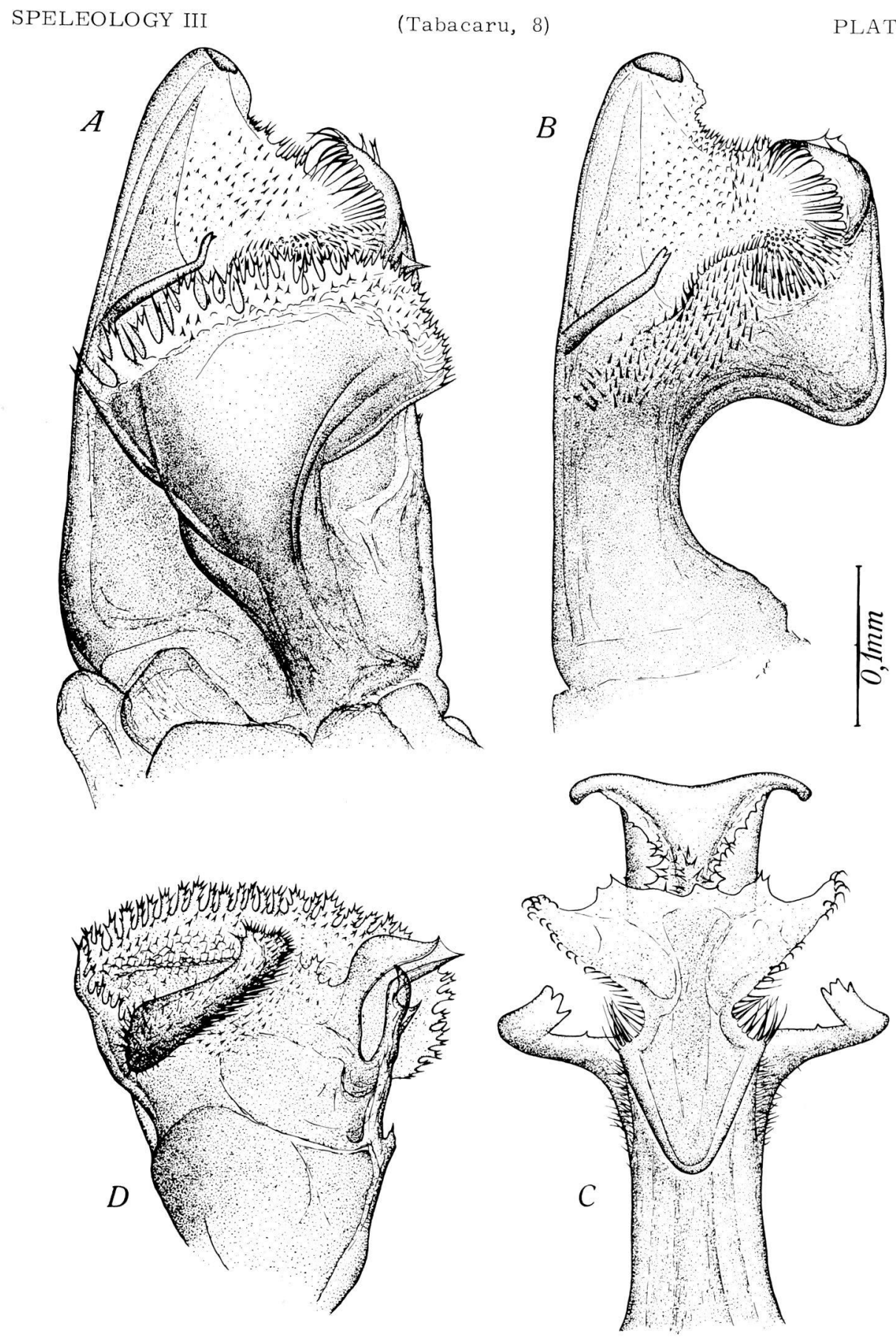

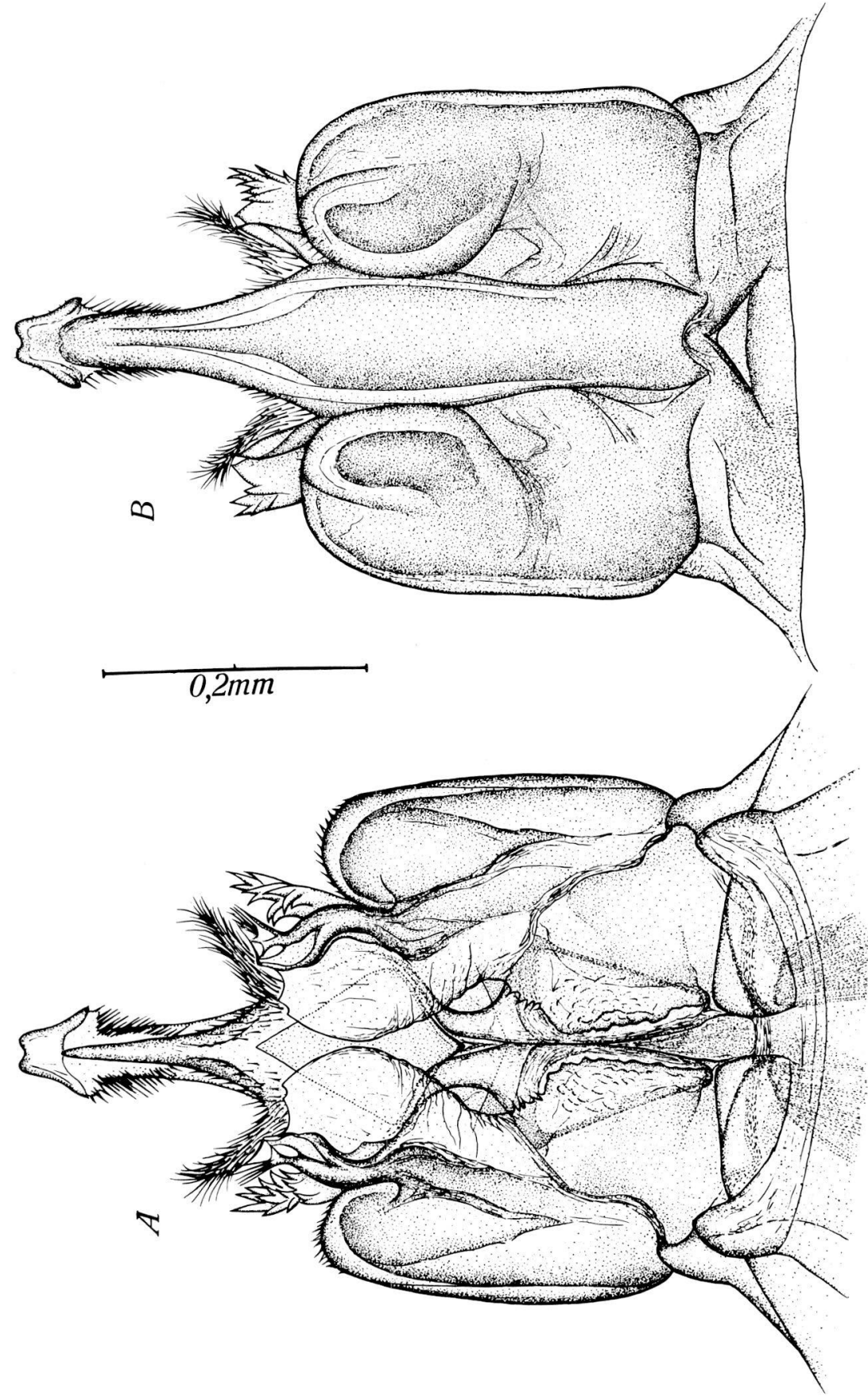

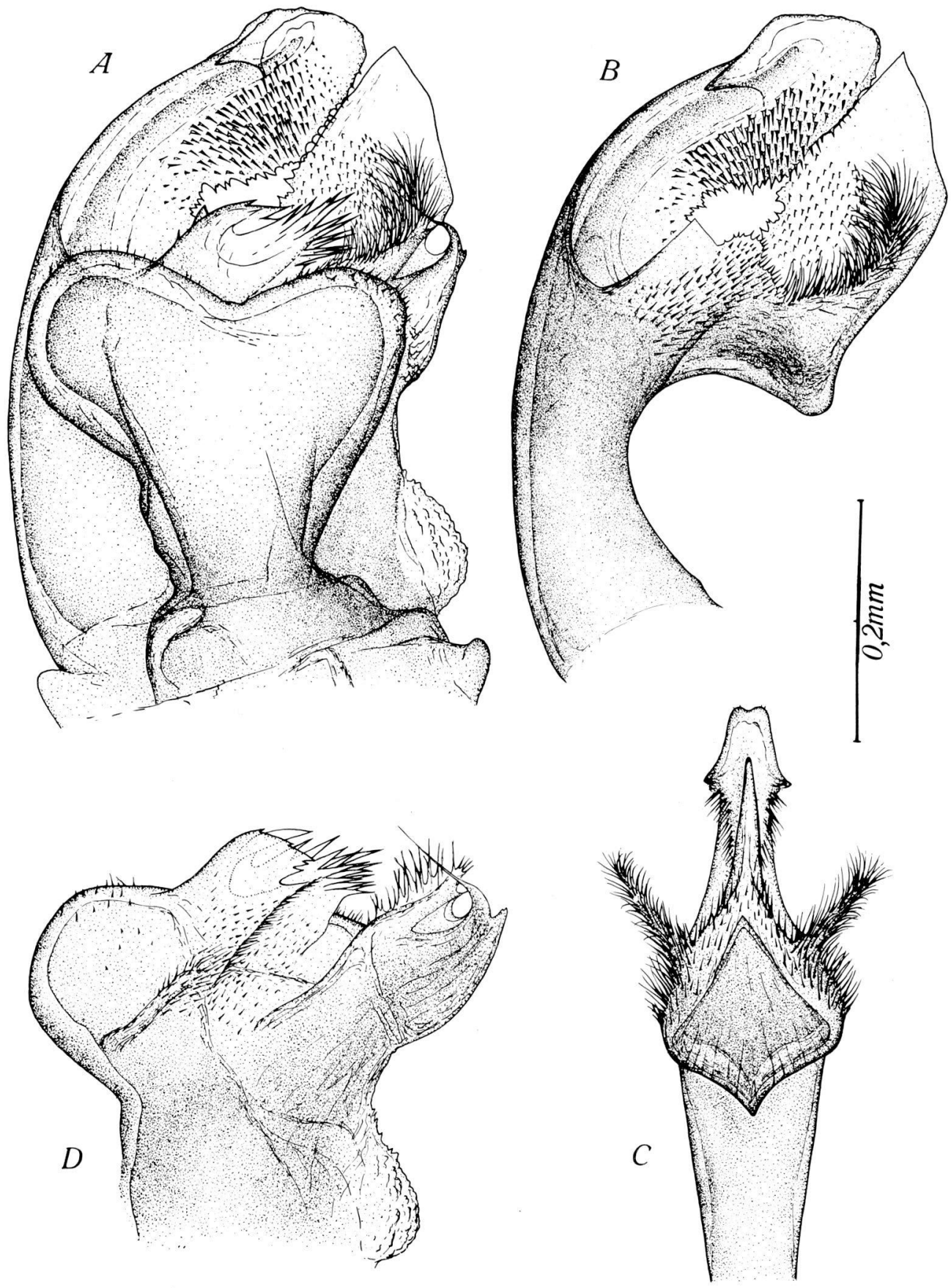

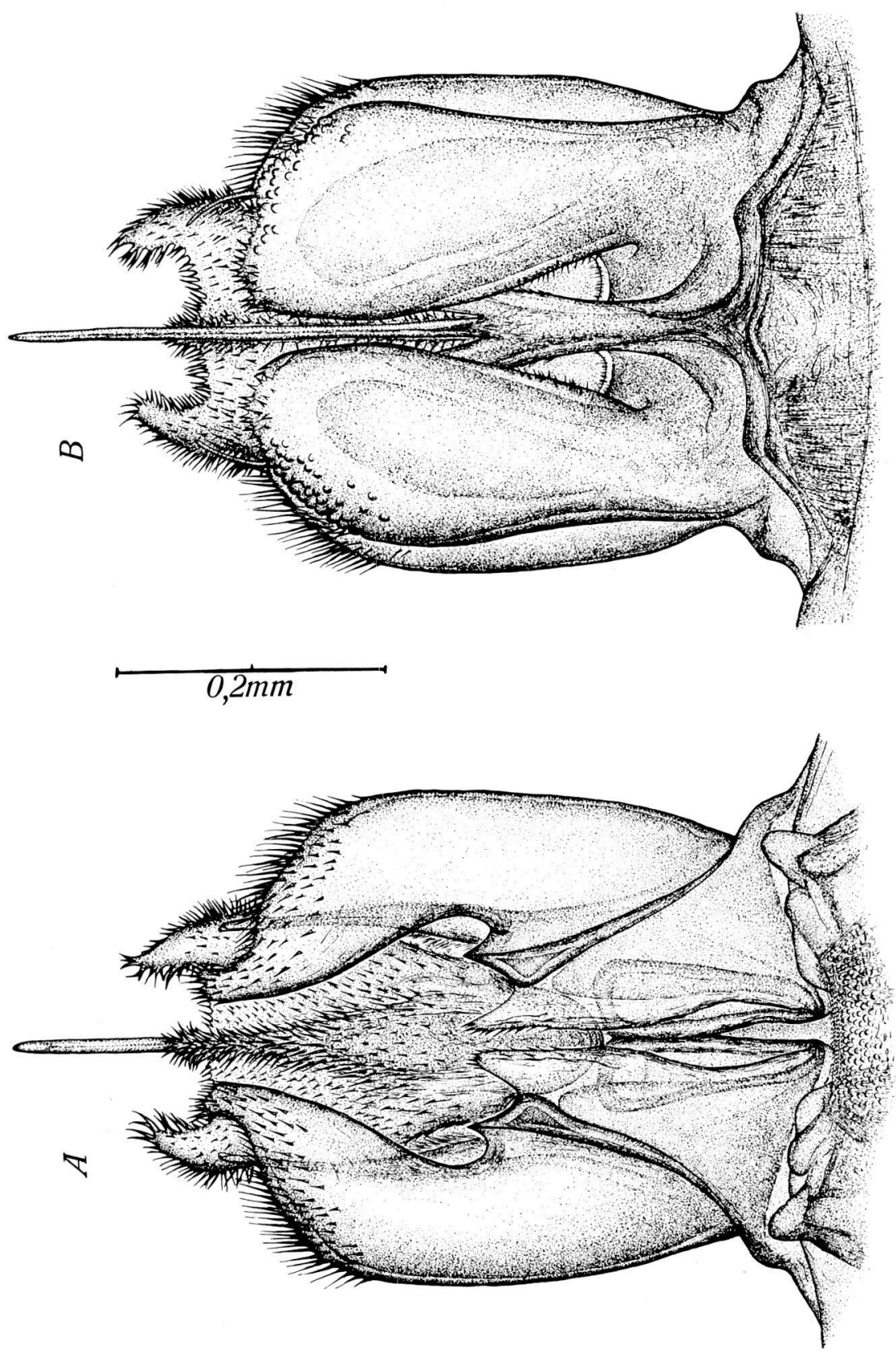

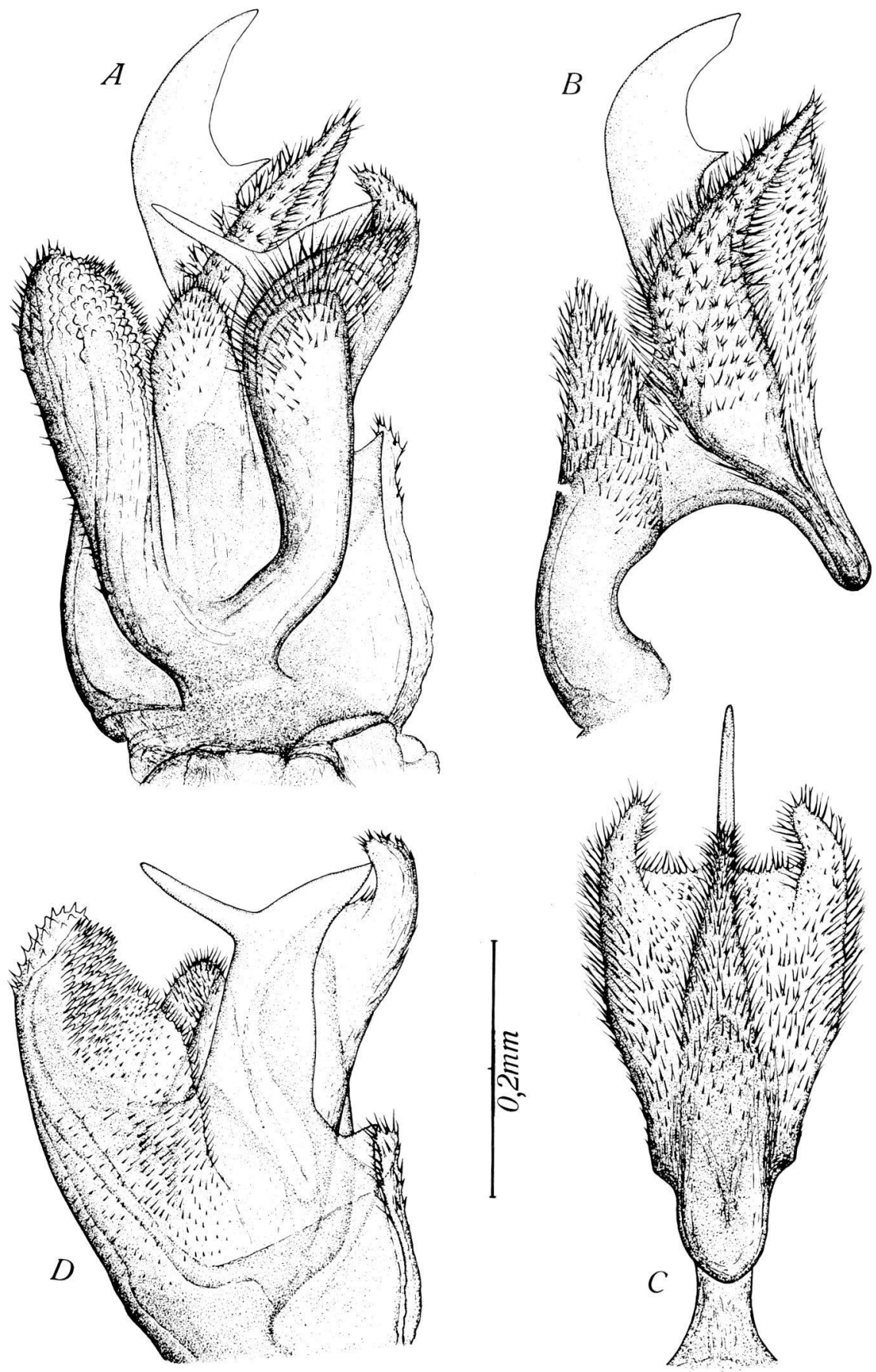


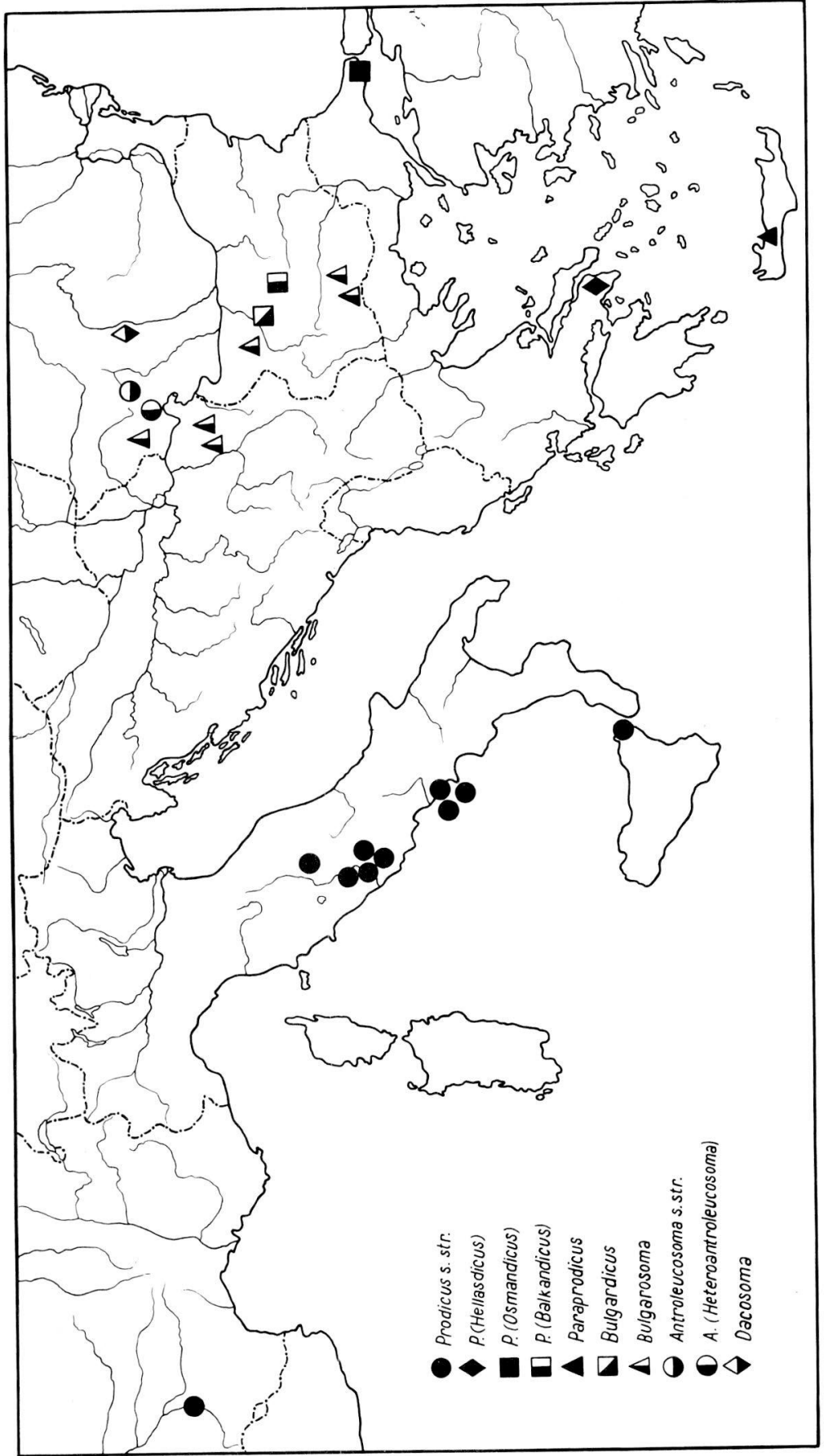

